IZA DP No. 6763

Measuring the Relative Productivity of Multitasking to Sole-tasking in Household Production: New Experimental Evidence

Gigi Foster

Charlene M. Kalenkoski

July 2012 


\title{
Measuring the Relative Productivity of Multitasking to Sole-tasking in Household Production: New Experimental Evidence
}

\author{
Gigi Foster \\ University of New South Wales \\ Charlene M. Kalenkoski \\ Ohio University \\ and IZA
}

Discussion Paper No. 6763

July 2012

IZA

P.O. Box 7240

53072 Bonn

Germany

Phone: +49-228-3894-0

Fax: +49-228-3894-180

E-mail: iza@iza.org

\begin{abstract}
Any opinions expressed here are those of the author(s) and not those of IZA. Research published in this series may include views on policy, but the institute itself takes no institutional policy positions.

The Institute for the Study of Labor (IZA) in Bonn is a local and virtual international research center and a place of communication between science, politics and business. IZA is an independent nonprofit organization supported by Deutsche Post Foundation. The center is associated with the University of Bonn and offers a stimulating research environment through its international network, workshops and conferences, data service, project support, research visits and doctoral program. IZA engages in (i) original and internationally competitive research in all fields of labor economics, (ii) development of policy concepts, and (iii) dissemination of research results and concepts to the interested public.
\end{abstract}

IZA Discussion Papers often represent preliminary work and are circulated to encourage discussion. Citation of such a paper should account for its provisional character. A revised version may be available directly from the author. 


\section{ABSTRACT}

\section{Measuring the Relative Productivity of Multitasking to Sole-tasking in Household Production: New Experimental Evidence*}

We present a household production model that incorporates multitasking and results from a customized experiment designed to measure the individual-specific productivity parameters from this model. We observe these productivity parameters under alternative incentive scenarios, designed to mimic changes in the relative utility payoffs to the two tasks. Using information on demographic and other characteristics collected through an on-screen survey taken by our experimental participants, we examine the correlates of individuals' sole-tasking and multitasking performance. Finally, we provide the literature's first measures of the ownand cross-price elasticities of household task supply in a multitasking context.

JEL Classification: D13, C91

Keywords: experiment, household production, child care, time use, productivity measures

Corresponding author:

Charlene Marie Kalenkoski

Ohio University

Department of Economics

Bentley Annex 351

Athens, $\mathrm{OH} 45701$

USA

E-mail:kalenkos@ohio.edu

\footnotetext{
* We thank Xi Mao and Markus Schaffner for research assistance. We are also grateful to the Australian School of Business Small Projects grant fund and the ASBLab at the University of New South Wales for enabling our experiments, and to seminar participants at UNSW and the University of Queensland for helpful suggestions. This research has the approval of the UNSW Human Research Ethics Committee. All errors and viewpoints are our own.
} 


\section{Introduction}

Child quality is one of the most important outcomes produced by households and much of it is produced using multitasked time. Both Floro \& Miles (2003) and Kalenkoski \& Foster (2008) use time-diary data to report on the prevalence and correlates of multitasked time. Floro \& Miles (2003) examine the incidence and determinants of time spent in 'overlapped' work activity, including household work, and find that gender, household life cycle and composition, education, cultural norms, employment status, and income influence the extent to which individuals (particularly women) perform these multitasked work activities. Kalenkoski \& Foster (2008), whose focus is parental child care time, regress sole-tasked and multitasked child care time on an even more exhaustive list of demographic and other explanatory variables, and find significant differences in the correlates of each. From an economic standpoint, these systematic patterns suggest that the choice to multitask may depend on individuals' ability to multitask different activities (i.e., their underlying multitasking productivity parameters for different activities) and/or their relative preferences over the outcomes produced by those different activities. However, time-diary data collection efforts usually do not involve the collection of information on the outcomes generated by various uses of time, so we cannot use typical time-diary data sets to estimate the relative productivity of multitasked versus sole-tasked time spent on household tasks. Therefore, in this paper we introduce a new experiment that is guided by theory and designed to enable us to directly observe and quantitatively compare participants' productivity in sole-tasked and multitasked household production.

To our knowledge, only one other study in economics has sought to measure multitasking productivity in an experimental setting. ${ }^{1}$ Buser \& Peter (2012) present an experiment where participants engage in two problem-solving tasks: Sudoku and word search puzzles. In different treatments the participants face sequential sole-tasking scenarios, are forced to move back and forth between tasks at pre-set intervals, or may choose whether or not to switch between tasks. These authors find that participants are significantly less productive when forced to switch between tasks than when forced to perform tasks sequentially. They also find, perhaps surprisingly, that when allowed to structure their own work (i.e., when allowed to decide whether to stay with a given task or switch to another at any given moment), participants are less productive than when forced to perform tasks sequentially. The average estimated decrease in task productivity across participants that the authors

\footnotetext{
${ }^{1}$ Experimental studies of multitasking - typically analyzed as part of investigations into 'task-switching' performance and its cognitive correlates - are abundant in psychology; see, for example, Rubinstein, Meyer \& Evans (2001).
} 
report when participants were forced to task-switch rather than stay with the one task is 7 to 11 percent. $^{2}$ Further, these authors directly test whether there is a difference between genders in the degree to which participants' productivity decreases in the task-switching stage compared to the single-task stage. Contrary to their priors, they find no significant gender difference.

The tasks used in the Buser \& Peter (2012) study are very different from the types of tasks that are typically multitasked in the home. In addition, their experiment does not mimic the situation in which much multitasking in the home is performed, that is, where one of the two tasks (child care) must be attended to most, if not all, of the time. Yet, the household setting is not only a hub of multitasking but also the main context in which nonmarket economic production occurs. Abraham \& Mackie (2005), Folbre (2006), and others have called for better measurement of such household production. Indeed, Folbre (2006) laments the lack of good data on the multitasking of child care. For these reasons, we argue that a productivity measure specific to household production that relates sole-tasking to multitasking productivity is needed.

We present results from a new experiment that enables us to directly observe the outcomes of sole-tasked and multitasked time use and to quantitatively measure participants' productivity in stylized versions of two sole-tasked and multitasked household production activities: baby care and clothes sorting. We examine true multitasked settings, settings in which two tasks demand simultaneous attention, unlike the task-switching scenarios studied in Buser \& Peter (2012). In order to determine the effect of differential preferences for the two outcomes, we manipulate the relative payoffs that participants receive for the different tasks. In addition, because our experimental participants complete a questionnaire capturing demographic, household, experiential, and psychological characteristics, we also examine the correlates of individuals' productivity parameters using standard regression analysis.

\section{Model}

The standard household production model pioneered by Gary Becker (Becker 1965) does not allow time to be spent simultaneously in different activities. From a conceptual perspective, this is problematic if an important activity cannot be started and ended discretely, which is true in the case of parental child care. As noted by Folbre, Yoon, Finnoff \& Fuligni

\footnotetext{
${ }^{2}$ This range, while not reported explicitly by the authors, is the result of our calculations based on Table 3 of their paper.
} 
(2005), Folbre \& Yoon (2007) and others, children cannot be 'turned off' in order for their parents to do other things. Yet, if a mother supervises her child while doing the laundry, her choice to multitask could only be mapped into the Beckerian framework if a fraction of the total minutes spent in this combined activity were allocated to child care and the rest were allocated to doing the laundry.

In Becker's original household production model (Becker 1965), individuals choose time spent in market work and household production to maximize utility subject to production functions for household commodities, a budget constraint, and a time constraint. In this model, a unit of time may be spent in only one activity.

We present a model that is similar in spirit to Becker's model but that focuses solely on the decision to sole-task or multitask household production activities. In this model, utility is represented by

$$
U=\alpha C+\beta H
$$

where individuals obtain utility from a child good, C, and a household good, H. $\alpha$ and $\beta$ are preference parameters. Specifically, the larger is the ratio of $\alpha$ to $\beta$, the more that the individual benefits from production of the child good relative to production of the household good.

The child-good production function is given by

$$
C=\gamma_{1} \ln t_{\mathrm{SC}}+\gamma_{2} \ln t_{\mathrm{M}}
$$

where $t_{S C}$ is sole-tasked time spent by the individual in production of the child good, $t_{M}$ is multitasked time spent by the individual in production of both goods, $\gamma_{1}$ is the productivity factor for sole-tasked time in producing the child good, and $\gamma_{2}$ is the productivity factor for multitasked time in producing the child good.

The analogous household-good production function is

$$
H=z_{1} \ln t_{\mathrm{SH}}+z_{2} \ln t_{\mathrm{M}}
$$

where $t_{S H}$ is sole-tasked time spent by the individual in household production, $z_{1}$ is the productivity factor for sole-tasked time in producing the household good, and $z_{2}$ is the productivity factor for multitasked time in producing the household good.

Our primary estimation targets in this paper are the baseline productivity parameters of housework and child production, $\gamma_{1}, \gamma_{2}, z_{1}$, and $z_{2}$, for each experimental participant, 
which are calculated as follows:

$$
\begin{aligned}
\gamma_{1} & =[\text { quantity of } C \text { produced in sole-tasked context }] / \ln \left(t_{\mathrm{SC}}\right) \\
\gamma_{2} & =[\text { quantity of } C \text { produced in multitasked context }] / \ln \left(t_{\mathrm{M}}\right) \\
z_{1} & =[\text { quantity of } H \text { produced in sole-tasked context }] / \ln \left(t_{\mathrm{SH}}\right) \\
z_{2} & =[\text { quantity of } H \text { produced in multitasked context }] / \ln \left(t_{\mathrm{M}}\right)
\end{aligned}
$$

Because we observe the quantities of output produced in our experimental setting ( $C$ and $H$ ), and because as the experimenters we also control (and therefore know) the time allocated to each production context, we can directly calculate these productivity parameters for each person. We also run regressions of our measured productivity parameters on an array of individual characteristics to examine their correlates. Our manipulation of the relative monetary payoffs to child care and housework in different experimental treatments mimics a change in the relative payoffs ( $\alpha$ and $\beta$ in our model) from producing the child good and the household good. We use our experimental manipulation of relative payoffs to estimate how the allocation of time within the multitasking setting differs when returns to the two tasks change. We interpret participants' responses to this manipulation as price elasticities of task supply.

\section{Experimental design}

Our experiment has several stages. The first and last stages are spent collecting various demographic, experiential, psychological, preference, and beliefs data from the participants. The rest are five income-earning stages that are presented in two different orders: one order for each of two groups of participants. These stages consist of (1) a sole-tasked baby care stage, lasting for three minutes; (2) a sole-tasked clothes-sorting stage, with a payoff regime likely to result in a lower total payoff than from the sole-tasked baby-care stage, lasting for three minutes; (3) a sole-tasked clothes-sorting stage, with a payoff regime likely to result in a higher total payoff than from the sole-tasked baby-care stage, lasting for three minutes; (4) a multitasked stage in which the clothes-sorting task is remunerated at the lower level, lasting for six minutes; (5) a multitasked stage in which the clothes-sorting task is remunerated at the higher level, lasting for six minutes.

In the pilot and the first four experimental sessions, the income-earning stages appear in exactly the order laid out above: sole-tasked baby care, sole-tasked clothes sorting (low payoff), sole-tasked clothes sorting (high payoff), multitasking (low payoff to clothes sorting), multitasking (high payoff to clothes sorting). In the final three experimental 
sessions, the stages involving a high-payoff clothes-sorting task appear before the stages involving a low-payoff clothes-sorting task. Hence, the order for these sessions is: soletasked baby care, sole-tasked clothes sorting (high payoff), sole-tasked clothes sorting (low payoff), multitasking (high payoff to clothes sorting), multitasking (low payoff to clothes sorting). Participants earn experimental dollars in accordance with their performance in each task, and earnings in the multitasked stages are completely independent across tasks.

Extensive testing was performed on this design in advance of the main round of the experiment, including two pilot runs. ${ }^{3}$ The goal of this testing was mainly to calibrate the relative payoffs to baby care and sorting clothes such that the money value of performance in the two tasks was roughly equivalent at a baseline level of remuneration for the clothessorting task. We then chose our two specific payoff regimes to bound that rough equivalence, such that in the low-payoff regime, it is generally more remunerative to increase effort on the baby care task when multitasking, while in the high-payoff regime, it is generally more remunerative to increase effort on the clothes-sorting task when multitasking.

Before all sole-tasked stages of the experiment, detailed on-screen instructions are presented regarding how to maximize one's payoff from the particular task. We also include instruction screens before every multitasked stage, reminding participants of the task objectives and the payout structure. Finally, to ensure that participants are as informed as possible about their relative performance on the different tasks throughout the experiment, on the instruction page at the start of each income-earning stage after the second, we inform participants of the relative payoffs they have earned in the prior stage or stages. On the information sheet appearing before the third stage, participants are informed of the ratio of their baby-care payoff to their first sole-tasked, clothes-sorting payoff; and on the information sheet preceding the fourth stage, they are informed of the ratio of their baby-care payoff to their second sole-tasked, clothes-sorting payoff. On the information sheet preceding the fifth and final income-earning stage, participants are told the ratio of their earnings in the baby care task to their earnings in the clothes-sorting task during the previous (multitasked) stage. Information about productivity in previous rounds is provided in order to ensure that participants are fully aware of the consequences of their actions during the income-earning stages.

Finally, in order to minimize the potential for wealth effects to contaminate effort choices and hence our measures of productivity, each participant's actual take-home payment from the entire experiment is calculated as the sum of his earnings in two randomly-selected, sole-

\footnotetext{
${ }^{3}$ Data from the second pilot are included in the analysis in this paper because neither the design nor the payoff schemes were changed between that pilot and the main rounds.
} 
tasked stages, plus his earnings in one randomly-selected, multitasked stage. ${ }^{4}$ Information about the payoff structure is clearly provided at the start of the experiment, along with a full description of the stages of the experiment, and ample time is allocated for participants' questions to be posed and answered by us.

\subsection{Baby care}

In the baby-care task, the participant is first shown a picture of a happy baby and presented with an icon showing a pacifier, which must be clicked when it appears - at random positions on the screen, which change at two-second intervals - in order to keep the baby in that happy mood. If the participant fails to click the pacifier button regularly enough, then the baby's mood will start to decline, following a random-walk process with a downward trend. As the baby's mood declines, pictures of successively less happy babies appear to the participant, and unpleasant sounds in the form of increasingly insistent baby cries are streamed through the headphones, which are compulsory for participants to wear.

Remuneration for this task, both in the sole-tasked and multitasked stages of the experiment, works in the following way. For each two-second interval that the baby's mood is at the highest level, the participant receives two experimental dollars. For each two-second interval that the baby's mood is at the next-highest level, the participant receives one experimental dollar. For each two-second interval that the baby's mood is at the secondto-worst level, the participant receives fifty experimental cents. Finally, the participant receives no compensation for any two-second interval during which the baby's mood is at the lowest level.

\subsection{Sorting clothes}

In the clothes-sorting task, the participant is confronted with an overlapping set of icons representing a never-ending pile of laundry of three types: men's white shirts, men's colored shirts, and ladies' blouses. The participant's task is to drag and drop each of these distinct types of icons into the appropriate wash-basket icon: the hot-wash basket (for men's white shirts), the warm-wash basket (for men's colored shirts), or the hand-wash basket (for

\footnotetext{
${ }^{4}$ Real-dollar earnings are calculated by using a fixed exchange rate relating experimental dollars to real dollars, where that rate was chosen to result in the average real-dollar payout being roughly equivalent to the ASBLab's standard payment of $\$ 15$ to $\$ 20$ per hour of participant time. Earnings calculated in this way from the income-earning stage are paid in addition to a $\$ 5$ show-up fee, which is paid to all participants, regardless of their performance.
} 
ladies' blouses).

For each shirt correctly (incorrectly) sorted, the participant earns (loses) two experimental dollars in the low-payoff regime or four experimental dollars in the high-payoff regime.

\subsection{Experimental infrastructure}

The experimental sessions reported in this paper were run in September 2011 in the ASBLab at the University of New South Wales. ORSEE (Greiner 2004) was used to recruit participants from a standing subject pool, consisting mainly of university students enrolled in study programs administered by the Australian School of Business. No participant exclusion criteria were applied during recruitment other than standard exclusions (no children, and no conflicts of interests) required by the UNSW Human Research Ethics Committee. The hard-copy instructions given to participants, and the first screen they faced when commencing the experiment, are reproduced in the Appendix.

\section{Analytical approach}

Using our experimental data, we can calculate each participant's productivity parameter on each type of time input (sole-tasked baby care $\left(\gamma_{1}\right)$, multitasked baby care $\left(\gamma_{2}\right)$, soletasked clothes sorting $\left(z_{1}\right)$, and multitasked clothes sorting $\left(z_{2}\right)$ ) using the formulas shown in Equations 1 through 4 in Section 2. Given our experimental design, $t_{S C}, t_{S H}$, and $t_{M}$ in these formulas are respectively the total minutes spent in sole-tasked baby care (i.e., three), the total minutes spent in sole-tasked clothes-sorting (three), and the total minutes spent in the multitasked context (six). $C$ is the weighted sum of time that the participant's actions kept the baby in the highest, next-highest, and third-highest moods, where the weights are identical to those used in the baby-care payoff function described above, i.e.,

$.5 *$ (number of two-second intervals during which baby was in second-lowest mood)

$+1 *$ (number of two-second intervals during which baby was in second-highest mood) $+2 *$ (number of two-second intervals during which baby was in highest mood),

and $H$ is simply the number of correctly-sorted shirts minus the number of incorrectlysorted shirts.

In addition to calculating and analyzing the raw productivity factors in isolation, we also calculate the ratios of $\gamma_{2}$ to $\gamma_{1}$ and $z_{2}$ to $z_{1}$ and interpret these ratios as measures of 
each participant's preservation of task productivity when s/he moves from a sole-tasking setting to a multitasking setting, for $C$ and $H$ respectively. This analysis - and all empirical analysis in the paper - is performed using the information from only one payoff regime per person, because we discard the data from the payoff regime in force during the participant's first sole-tasked clothes-sorting stage and first multitasked stage due to concerns about learning effects. We expect that people's task performance will improve as they acquire experience doing the tasks, and we do not wish to compare people's productivity the first time they perform the clothes-sorting task, or the first time they multitask in our experiment, with their productivity in later stages. ${ }^{5}$

After a descriptive analysis of our measured productivity factors themselves, we regress these factors as well as their ratios on the set of control variables discussed in the next section in order to identify their correlates.

Finally, we aggregate the data from the non-learning stages faced by each participant to construct estimates of effort price elasticities for both types of tasks. We first construct the average per-minute output of sole-tasked and multitasked time in the baby-care task and the clothes-sorting task for each participant, under whichever of the two different payoff regimes was in place during the non-learning stages for that participant. We then calculate the means of these 'average products' across participants, and use them to construct price elasticities of task supply in a multitasked context.

\subsection{Control variables}

We collected data on standard demographics as well as a wide variety of other variables for each participant. A full list of the questions asked appears in the Appendix. Because we are agnostic about which variables are most or least likely to be influential in explaining our productivity parameters, we include in our simple regression models a wide variety of control variables that satisfy at least one of three criteria: they are economically meaningful; they control for experiential or preference heterogeneity across people that could plausibly be important in this application; and/or they were statistically significant in our preliminary tests.

The variables we include are described thoroughly in Tables 1a and 1b. The types of

\footnotetext{
${ }^{5}$ The sole-tasked baby care stage is extremely easy to master, as all that is involved is clicking on a button repeatedly when it appears. By contrast, the sole-tasked clothes-sorting task does require that participants learn how to drag and drop the icons, and that they accurately match clothes to the correct piles. Therefore, we only anticipate (and in practice, we only see) a sole-tasking learning effect for clothes sorting.
} 
variation they capture are briefly as follows (with relevant variable nicknames following in italics): gender (female); culture with which the participant identifies most strongly (oth_asian, pan_asian); marital status (married); age (age); personal weekly after-tax income (inc1 through inc5); family wealth (famwealth2 and famwealth3); occupation class of the participant's mother (mumocc1 through mumocc5) and father (dadocc1 through dadocc4); completed schooling of the participant's mother (mumschool1 through mumschool3) and father (dadschool1 through dadschool3); program of study (commerce_d); whether the participant enjoys being busy (enjoy), believes himself to be good at multitasking (goodmt), plays computer games frequently (game), feels things get done better when they are done one at a time (judge_mt), or frequently played caretaking games with dolls or stuffed animals as a child ( $d o l l)$; number of siblings (siblings); whether the participant reports that he gets bored easily (boredeasy), frequently has time he doesn't know what to do

with (sparetime), or frequently multitasks (freqmult); the degree of stress the participant reports experiencing in the sole-tasked child care (stresscc), sole-tasked clothes sorting (stresshw), and multitasked (stressmult) stages in the experiment; the participant's reported political leanings (political); the range of hours the participant reports having spent with young children (hrswithkid2 through hrswithkid4) and having been solely responsible for young children (hrsresponsible2 through hrsresponsible 4); self-esteem (self_esteem); locus of control (loc_full); overall happiness (happy3 through happy5); self-perceived level of performance at university ( perfuni1 through perfuni3); left-handedness (lefthand); whether the participant had previously participated in an experiment at the ASBLab (experiment); and the experimental session in which the participant participated, which is controlled using a simple set of dummies.

\section{Experimental results}

\subsection{Individual productivity parameters}

We first calculate the productivity parameters associated with sole- and multitasked time for each good for each participant using the formulas in Equations 1 through 4, performing calculations only for the non-learning stages faced by each participant. Histograms for the different productivity parameters are displayed in Figures $1\left(\gamma_{1}\right), 2\left(\gamma_{2}\right), 3\left(z_{1}\right)$ and $4\left(z_{2}\right)$, separately by payoff regime.

The empirical ceiling clearly evident in Figure 1 indicates that our sole-tasked babycare productivity parameter is likely to be an underestimate for many participants, as the vast majority of people performed perfectly on the baby task by keeping the baby in the 
Table 1a: Full Covariate Definitions: Economic and Standard Demographic Variables

\begin{tabular}{|c|c|}
\hline Variable Name & Full Definition ('pt' stands for 'participant') \\
\hline female & 1 if $\mathrm{pt}$ is female; 0 otherwise \\
\hline c_othasian & $\begin{array}{l}1 \text { if pt identifies most strongly with culture of Hong Kong, Malaysia, or Singapore; } \\
0 \text { otherwise }\end{array}$ \\
\hline c_panasian & $\begin{array}{l}1 \text { if pt identifies most strongly with culture of Brunei, India, Indonesia, Japan, Pakistan, Korea, Taiwan, Vietnam, } \\
\text { non-specific "Asia", or "eastern culture"; O otherwise }\end{array}$ \\
\hline married & 1 if $\mathrm{pt}$ is married; 0 otherwise \\
\hline age & Chronological age of pt \\
\hline inc1 & $\begin{array}{l}1 \text { if pt reports his personal weekly after-tax income to be } \$ 100-\$ 199 \text {; } \\
0 \text { otherwise }\end{array}$ \\
\hline inc2 & $\begin{array}{l}1 \text { if pt reports his personal weekly after-tax income to be } \$ 200-\$ 299 \text {; } \\
0 \text { otherwise }\end{array}$ \\
\hline inc3 & $\begin{array}{l}1 \text { if pt reports his personal weekly after-tax income to be } \$ 300-\$ 399 \text {; } \\
0 \text { otherwise }\end{array}$ \\
\hline inc4 & $\begin{array}{l}1 \text { if pt reports his personal weekly after-tax income to be } \$ 400-\$ 499 \text {; } \\
0 \text { otherwise }\end{array}$ \\
\hline inc5 & $\begin{array}{l}1 \text { if pt reports his personal weekly after-tax income to be } \$ 500 \text { or more; } \\
0 \text { otherwise }\end{array}$ \\
\hline famwealth2 & $\begin{array}{l}1 \text { if pt reports his family to be 'the same' in terms of wealth as others; } \\
0 \text { otherwise }\end{array}$ \\
\hline famwealth3 & $\begin{array}{l}1 \text { if pt reports his family to be 'poorer' than others; } \\
0 \text { otherwise }\end{array}$ \\
\hline mumocc1 & $\begin{array}{l}1 \text { if pt's mother is a manager; } \\
0 \text { otherwise }\end{array}$ \\
\hline mumocc 2 & $\begin{array}{l}1 \text { if pt's mother is a professional; } \\
0 \text { otherwise }\end{array}$ \\
\hline mumocc3 & $\begin{array}{l}1 \text { if pt's mother is an associate professional; } \\
0 \text { otherwise }\end{array}$ \\
\hline mumocc 4 & $\begin{array}{l}1 \text { if pt's mother is a tradesperson, transport worker or laborer; } \\
0 \text { otherwise }\end{array}$ \\
\hline mumocc5 & $\begin{array}{l}1 \text { if pt's mother is some type of clerical worker; } \\
0 \text { otherwise }\end{array}$ \\
\hline dadocc 1 & $\begin{array}{l}1 \text { if pt's father is a manager; } \\
0 \text { otherwise }\end{array}$ \\
\hline dadocc2 & $\begin{array}{l}1 \text { if pt's father is a professional or associate professional; } \\
0 \text { otherwise }\end{array}$ \\
\hline dadocc3 & $\begin{array}{l}1 \text { if pt's father is a tradesperson, transport worker or laborer; } \\
0 \text { otherwise }\end{array}$ \\
\hline dadocc 4 & $\begin{array}{l}1 \text { if pt's father is some type of clerical worker; } \\
0 \text { otherwise }\end{array}$ \\
\hline mumschool1 & $\begin{array}{l}1 \text { if pt's mother received no post-high school degree, or if pt does not know } \\
\text { his mother's educational status; } 0 \text { otherwise }\end{array}$ \\
\hline mumschool2 & $\begin{array}{l}1 \text { if pt's mother received a post-high school qualification from a technical institute; } \\
0 \text { otherwise }\end{array}$ \\
\hline mumschool3 & $\begin{array}{l}1 \text { if pt's mother received a post-high school qualification from a university; } \\
0 \text { otherwise }\end{array}$ \\
\hline dadschool1 & $\begin{array}{l}1 \text { if pt's father received no post-high school degree, or if pt does not know } \\
\text { his father's educational status; } 0 \text { otherwise }\end{array}$ \\
\hline dadschool2 & $\begin{array}{l}1 \text { if pt's father received a post-high school qualification from a technical institute; } \\
0 \text { otherwise }\end{array}$ \\
\hline dadschool3 & $\begin{array}{l}1 \text { if pt's father received a post-high school qualification from a university; } \\
0 \text { otherwise }\end{array}$ \\
\hline
\end{tabular}


Table 1b: Full Covariate Definitions: Other Variables

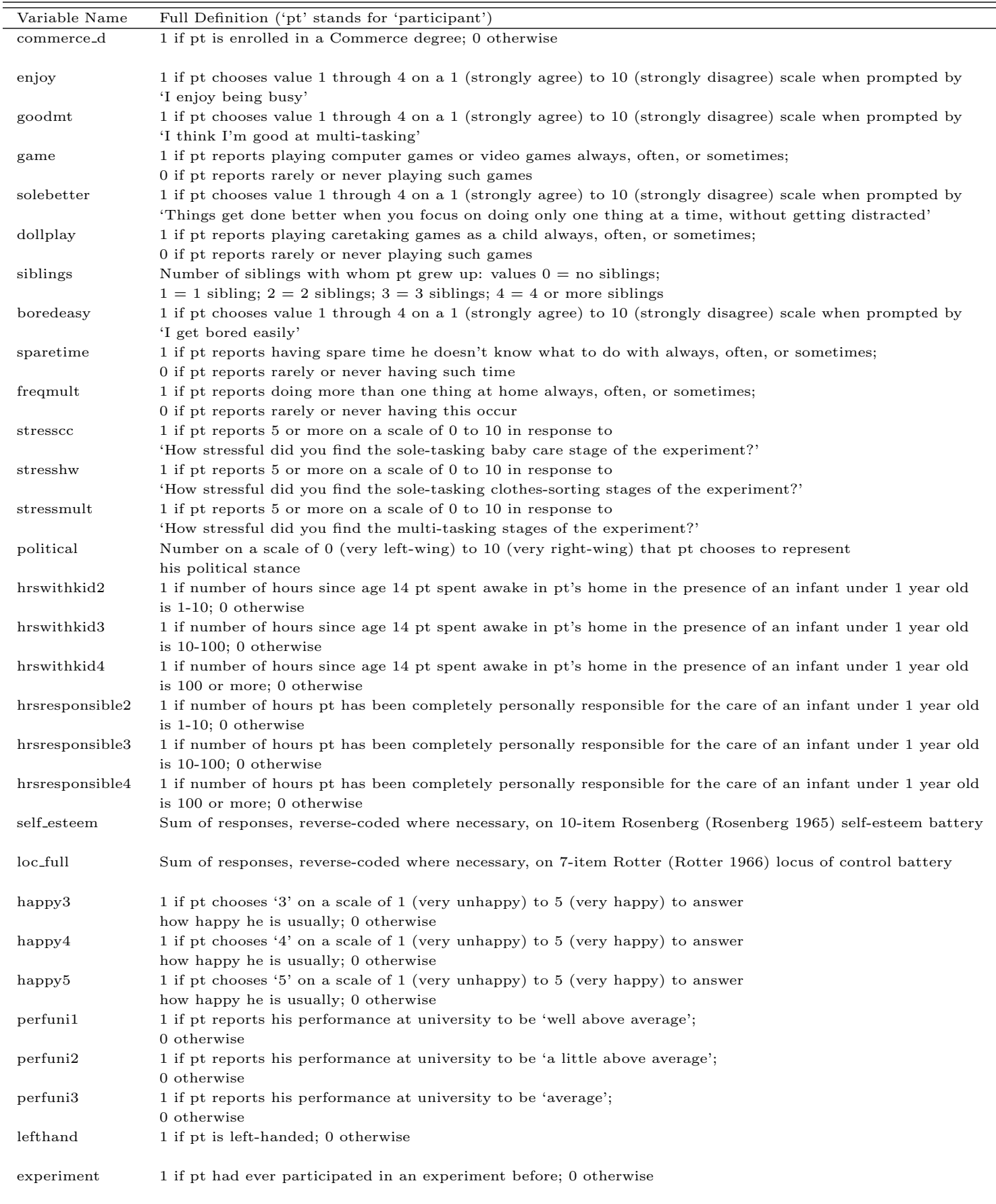




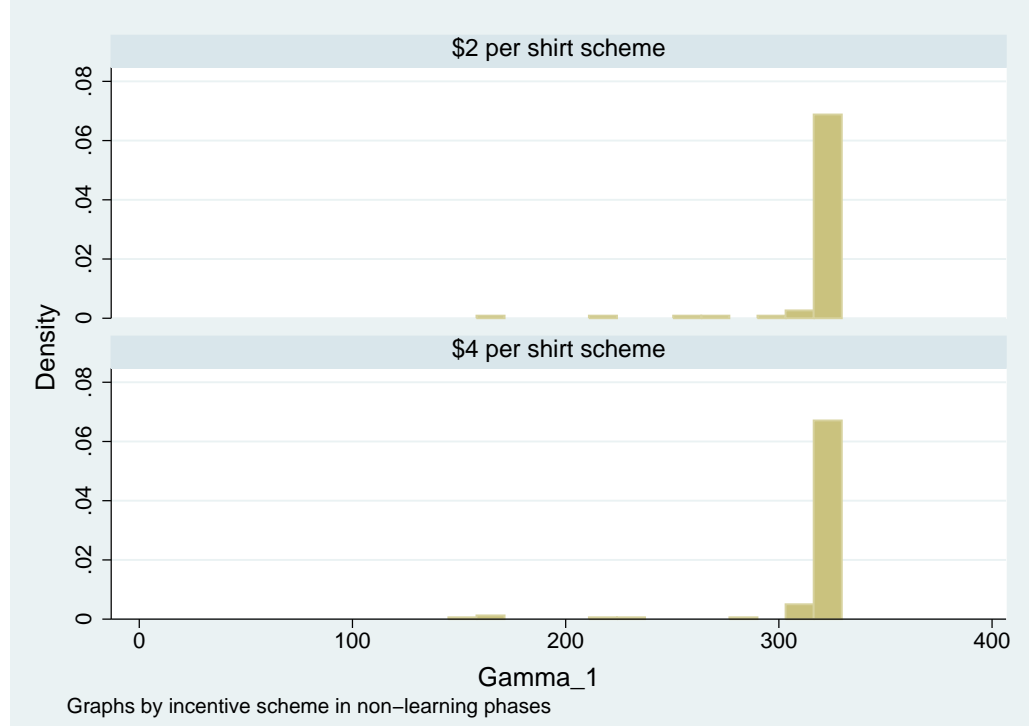

Figure 1: $\gamma_{1}$ (sole-tasking baby care productivity parameter): $\$ 2 /$ shirt scheme (top) versus $\$ 4 /$ shirt scheme (bottom)

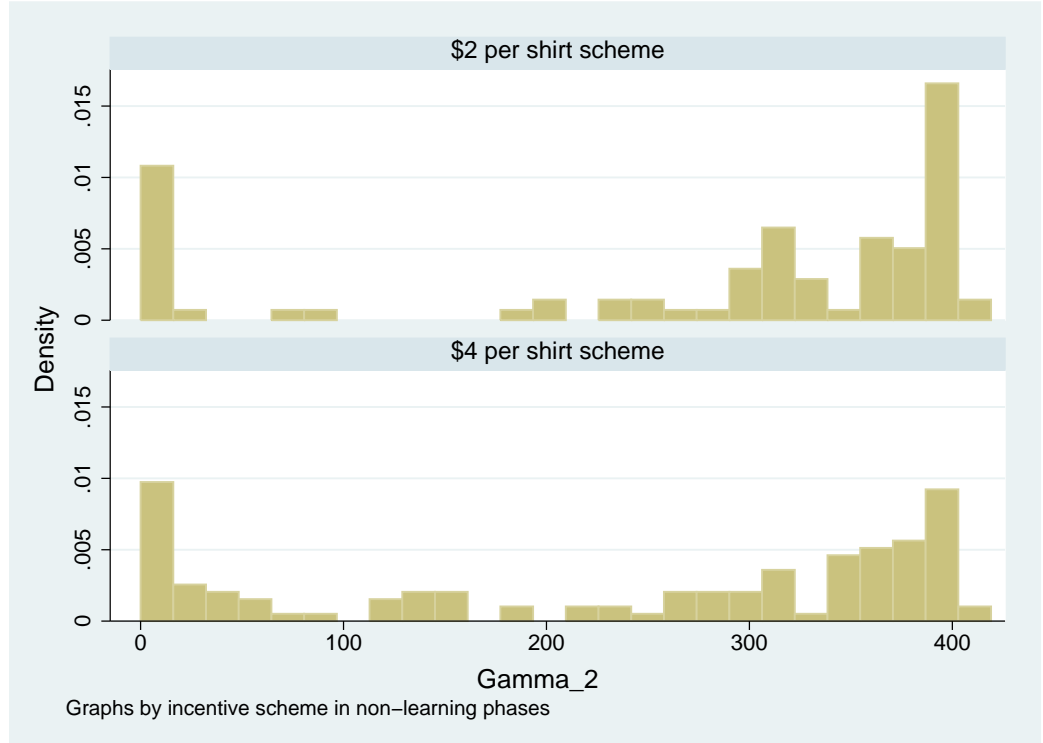

Figure 2: $\gamma_{2}$ (multitasking baby care productivity parameter): $\$ 2 /$ shirt scheme (top) versus $\$ 4 /$ shirt scheme (bottom) 


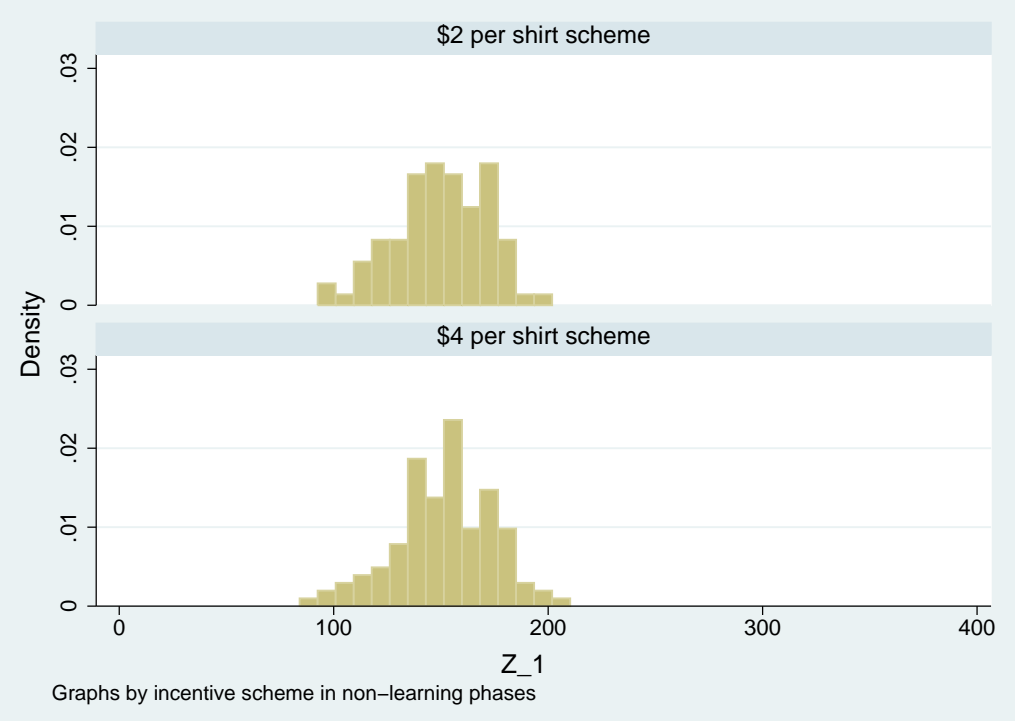

Figure 3: $z_{1}$ (sole-tasking clothes-sorting productivity parameter): $\$ 2 /$ shirt scheme (top) versus $\$ 4 /$ shirt scheme (bottom)

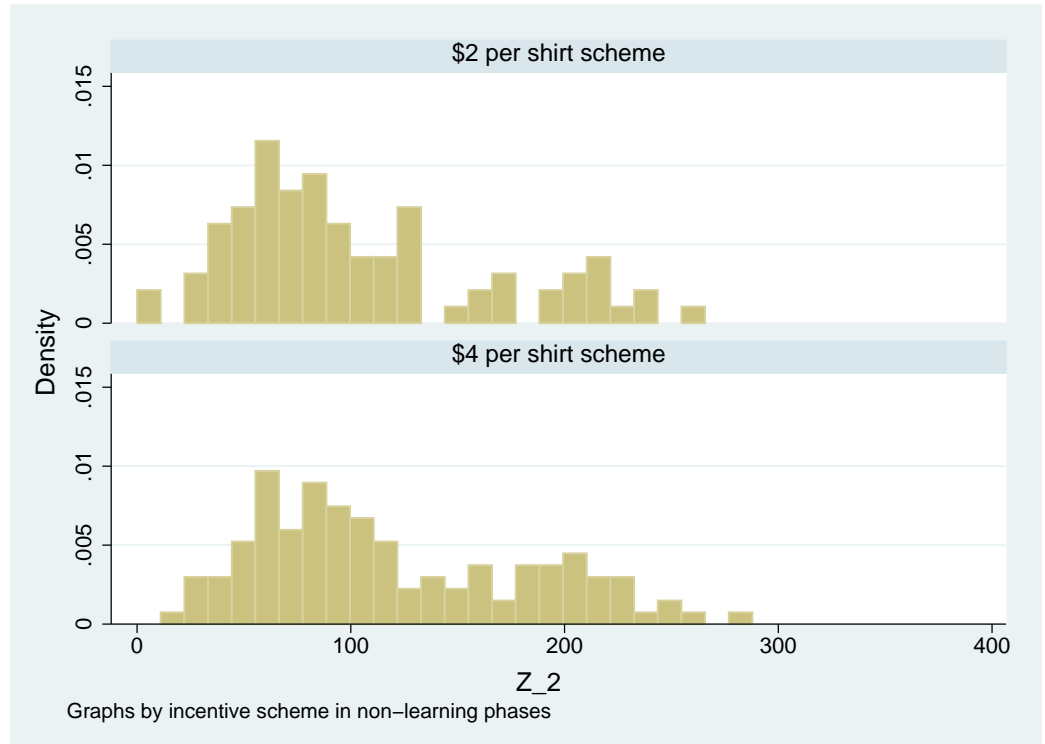

Figure 4: $z_{2}$ (multitasking clothes-sorting productivity parameter): $\$ 2 /$ shirt scheme (top) versus $\$ 4 /$ shirt scheme (bottom) 
happiest possible mood for the entire three minutes of sole-tasked baby-care time. Babycare productivity fell and became far more heterogeneous across people in the multitasked stages, as seen by comparing Figure 1 with Figure 2. Comparing the top and bottom panels of Figure 2 also reveals more density in the left-hand side of the bottom panel than the left-hand side of the top panel, underscoring that there was, on average, an additional loss in baby care productivity when the alternative task of sorting shirts was more highly remunerated. However, some participants still returned very high baby-care productivity in the multitasked setting, even when the alternative task was more highly rewarded.

Figure 3 shows that participants' productivity in sole-tasked clothes-sorting is not strongly affected by the payoff regime. The loss in productivity when participants move to a multitasked setting is shown clearly by comparing Figure 3 to Figure 4. Comparing the top and bottom panels of Figure 4 also shows that the density of individual clothes-sorting productivity parameters is shifted slightly rightward when the multitasking is performed in a setting in which clothes-sorting is more highly remunerated, following participants' material incentives.

Taken together, these figures indicate first that participants' multitasking productivity in each task is generally lower than their sole-tasking productivity in that task, and second that multitasking productivity in each task is affected by the incentives embedded in the relative payoffs available to the two tasks.

Table 2 shows descriptive statistics for the calculated productivity parameters. Note that the productivity parameters cannot be directly compared across the two tasks, given that the scale of the baby care productivity figures reflects the fact that baby care 'output' is calculated in terms of experimental dollars, whereas clothes-sorting output is calculated in terms of numbers of shirts sorted.

Panel A presents the parameters estimated under the $\$ 2$ per shirt incentive scheme, and Panel B presents the parameters estimated under the $\$ 4$ per shirt incentive scheme. In Panel A, therefore, baby care is relatively more remunerative than clothes sorting, whereas in Panel B, sorting clothes is relatively more remunerative than baby care. Note that neither of the sole-task productivity parameters $\left(\gamma_{1}\right.$ or $\left.z_{1}\right)$ changes very much across incentive schemes. This is to be expected, given that in the sole-task settings there are no competing tasks. However, the multitasking productivity parameters, $\gamma_{2}$ and $z_{2}$, do change as expected across incentive schemes. When sorting clothes becomes more remunerative relative to baby care, the measured multitasked productivity of baby care falls 15\%, from 279 to 237, whereas the multitasked productivity of sorting clothes increases by 17\%, from 102 to 119. Similarly, the ratio of multitasking productivity to sole-tasking productivity for baby 
care falls from 0.86 to 0.75 as baby care becomes relatively less remunerative, while the analogous ratio for clothes sorting increases from 0.68 to 0.77 .

Table 2: Productivity Parameters

\begin{tabular}{lccccc}
\hline \hline Panel A: \$2 per shirt scheme & & & & & \\
Variable & $\mathrm{N}$ & Average & Std Dev & Min & Max \\
\hline$\gamma_{1}$ (ST baby care) & 86 & 323.2355 & 23.02494 & 169.3045 & 329.5066 \\
$\gamma_{2}$ (MT baby care) & 86 & 278.919 & 143.5545 & 11.44127 & 402.9559 \\
$z_{1}$ (ST clothes-sorting) & 86 & 150.0413 & 21.50526 & 95.57512 & 193.881 \\
$z_{2}$ (MT clothes-sorting) & 86 & 102.4068 & 60.71167 & 3.348664 & 261.1958 \\
$\gamma_{2} / \gamma_{1}$ & 86 & .8646426 & .4477164 & .0347224 & 1.811422 \\
$z_{2} / z_{1}$ & 86 & .6759137 & .3838211 & .0238889 & 1.580191 \\
\hline \hline Panel B: $\$ 4$ per shirt scheme & & & & & \\
Variable & $\mathrm{N}$ & Average & Std Dev & Min & Max \\
\hline$\gamma_{1}$ (ST baby care) & 121 & 320.8518 & 29.29559 & 155.1958 & 329.5066 \\
$\gamma_{2}$ (MT baby care) & 121 & 236.5559 & 149.5199 & 11.44127 & 402.9559 \\
$z_{1}$ (ST clothes-sorting) & 121 & 151.9723 & 22.5721 & 91.93416 & 210.2653 \\
$z_{2}$ (MT clothes-sorting) & 121 & 118.7761 & 62.83237 & 18.97576 & 276.8229 \\
$\gamma_{2} / \gamma_{1}$ & 121 & .7505607 & .4963329 & .0347224 & 2.248755 \\
$z_{2} / z_{1}$ & 121 & .7712011 & .3696928 & .1248324 & 1.635059 \\
\hline \hline
\end{tabular}

Data from the participants in the final three experimental rounds is used to construct Panel A; data from participants in the pilot plus the first four experimental rounds is used to construct Panel B. Samples exclude a handful of records which demonstrated clear confusion about the task instructions (e.g., those earning zero dollars for any task). See text for full descriptions of experimental treatments.

\subsection{Observable correlates of the productivity parameters}

In Tables $3 \mathrm{a}$ and $3 \mathrm{~b}$, we explore the extent to which the productivity parameters discussed in the previous section are predictable using the demographic, psychological, and experiential variables that we observe. Due to the plethora of covariates, we split the presentation of these regression results into two tables. Table 3 a shows coefficient estimates on the 'economic' variables (such as income, family wealth, and parental background) and standard demographic variables (such as age and gender) that we use to predict each productivity parameter. Table $3 \mathrm{~b}$ then shows coefficient estimates on the other variables we are able to construct using our unique survey data. Both tables show fit statistics and observa- 
tion counts, but each column of Table $3 \mathrm{~b}$ is merely a continuation of the same regression results reported in that same column from Table 3a. Columns (1) and (2) of each table provide regressions of the productivity parameter $\gamma_{1}$ under the $\$ 2$ per shirt and $\$ 4$ per shirt schemes, respectively. Columns (3) and (4) then present regressions for $\gamma_{2}$ under the two incentive schemes. Columns (5) through (8) similarly provide results for $z_{1}$ and $z_{2}$. All variable names in Tables $3 \mathrm{a}$ and $3 \mathrm{~b}$ are explained in Tables $1 \mathrm{a}$ and $1 \mathrm{~b}$, respectively.

The most noticeable aspect of Tables $3 \mathrm{a}$ and $3 \mathrm{~b}$ is a striking change in explanatory power across columns, including even negative adjusted R-squared values in several columns despite the inclusion of a host of control variables. Of course, only a modest amount of explanatory power should be expected when the dependent variable is sole-tasking productivity in the production of the child good (i.e., Columns (1) and (2)), given that many individuals in the sample kept the baby completely happy for the entire duration of the sole-tasking stage. When we move to Column (3), predicting the multitasking baby-care productivity factor at the lower per-shirt remuneration level, the explanatory power jumps to over $50 \%$ and many independent variables become significant. Those participants who identify with the cultures of Hong Kong, Malaysia, or Singapore; who are married; who have mid-range personal income levels; whose families are reportedly poorer than others; whose mothers are managers; who are not enrolled in Commerce programs; who report high stress associated with the child care task or low stress associated with the clothessorting task; who have never been personally responsible for the care of a child; or who report themselves to be well above-average students have statistically significantly higher multitasking child care productivities at a $5 \%$ or lower p-value than other participants. This increase would most logically be offset by a lower productivity in the clothes-sorting activity. Indeed, when we predict multitasking housework productivity in the $\$ 2$ per shirt scheme in Column (7), that is just what we see for many of the same covariates. This compensating effect is evident for marital status, income, wealth, program of study, and stress associated with the child care task. For all other variables significant in Column (3), the compensating effects presented in Column (7) are in the correct direction but do not achieve statistical significance at the $5 \%$ level. We also note that many other variables' coefficient estimates in both columns are very large in magnitude but do not achieve statistical significance, probably due to modest sample size.

Our primary interpretation of the results in Columns (3) and (7) is that certain types of economic and cultural backgrounds predispose a person to spend more effort on the interactive baby-care task than on the menial clothes-sorting task when faced with the multitasking context and the lower payoff to clothes-sorting. Some people may simply be 
raised to place a particularly high value on the child good relative to the household good. Even in a setting in which the household good is almost as valuable in monetary terms as the child good, these people still follow their habits and spend more effort than others do on the baby, and less effort than others do on the household task.

The most interesting results in Tables $3 \mathrm{a}$ and $3 \mathrm{~b}$ relate to how the strength and significance of the explanatory variables change as we change incentive schemes in the multitasking context. Specifically, paying more per shirt in the multitasking setting (Columns (8) and (4) compared to Columns (7) and (3), respectively) appears to wipe out both explanatory power and statistically significant relationships, almost across the board. This suggests that apparent multitasking productivity differentials - which may arise through upbringing, genes, or habit - can be overcome with a change in economic incentives.

Sole-tasking productivity in the production of the household good (Columns (5) and (6)) is fairly well-predicted, though not as well as multitasking productivities in the $\$ 2$ per shirt scheme. $z_{1}$ is negatively associated with mid-range parental wealth, having a mother who works in a clerical occupation, having a mother with a university degree, and frequently feeling rushed. It could be that such individuals' more-advantaged upbringing may make it less necessary for them to acquire household production skills, or may lead them to place a lower absolute value on performing menial household-production tasks.

In Tables $4 \mathrm{a}$ and $4 \mathrm{~b}$, the ratios of productivity factors, $\gamma_{2} / \gamma_{1}$ and $z_{2} / z_{1}$, are the dependent variables, and the table structure is identical to that of Tables $3 \mathrm{a}$ and $3 \mathrm{~b}$. Under the $\$ 2$ per shirt incentive scheme, statistically significantly higher relative productivity of multitasked time in the production of the child good is coupled with statistically significantly lower relative productivity of multitasked time in the production of the household good for the those who identify with the cultures of Hong Kong, Malaysia, or Singapore; who are married; who have mid-range personal income levels; whose families are reportedly poorer than others; or who report high stress associated with the child care task. These results are consistent with the results in Tables $3 \mathrm{a}$ and $3 \mathrm{~b}$, and the other variables significant in those prior tables do show the expected signs in Tables $4 \mathrm{a}$ and $4 \mathrm{~b}$, though without statistical significance at the $5 \%$ level. Also consistent with our prior results, changing the payoff to $\$ 4$ per shirt removes all of these correlations and sends the explanatory power of the regressions into the negative range, suggesting that innate preferences for the child good in a multitasking context can be overcome with an increase in the relative payoff to housework. 
Table 3a: Correlates of Productivity Parameters: Economic and Standard Demographic Covariates

\begin{tabular}{|c|c|c|c|c|c|c|c|c|}
\hline \multirow[b]{3}{*}{ Scheme } & \multirow{2}{*}{\multicolumn{2}{|c|}{ (1) $-\gamma_{1}-$}} & \multirow{2}{*}{\multicolumn{2}{|c|}{ (3) }} & \multirow{2}{*}{\multicolumn{2}{|c|}{ (5) }} & \multirow{2}{*}{\multicolumn{2}{|c|}{ (7) }} \\
\hline & & & & & & & & \\
\hline & $\$ 2$ per shirt & $\$ 4$ per shirt & $\$ 2$ per shirt & $\$ 4$ per shirt & $\$ 2$ per shirt & $\$ 4$ per shirt & $\$ 2$ per shirt & $\$ 4$ per shirt \\
\hline \multirow[t]{2}{*}{ female } & -0.282 & -9.411 & -62.947 & -13.242 & 7.449 & -2.180 & 15.260 & -8.289 \\
\hline & $(22.14)$ & $(6.56)$ & $(83.40)$ & $(55.16)$ & $(15.54)$ & $(6.28)$ & $(40.37)$ & $(22.42)$ \\
\hline \multirow[t]{2}{*}{ c_othasian } & 39.530 & 3.787 & $390.993^{*}$ & $173.449^{*}$ & 41.437 & 1.301 & -127.592 & -49.743 \\
\hline & $(39.45)$ & $(8.75)$ & $(148.59)$ & $(73.50)$ & $(27.68)$ & $(8.37)$ & $(71.92)$ & $(29.87)$ \\
\hline \multirow[t]{2}{*}{ c_panasian } & 21.919 & -2.706 & 244.908 & 31.256 & -17.431 & -11.696 & -60.754 & -29.024 \\
\hline & $(33.11)$ & $(9.13)$ & $(124.71)$ & $(76.77)$ & $(23.23)$ & $(8.74)$ & $(60.36)$ & $(31.20)$ \\
\hline \multirow[t]{2}{*}{ married } & 74.195 & -50.635 & $926.628^{*}$ & 401.205 & -41.781 & -45.042 & $-374.532 *$ & -186.789 \\
\hline & $(80.75)$ & $(30.89)$ & (304.18) & $(259.60)$ & $(56.66)$ & $(29.56)$ & $(147.23)$ & $(105.50)$ \\
\hline \multirow[t]{2}{*}{ age } & -2.324 & 0.894 & -41.732 & -1.841 & 2.421 & $3.336^{*}$ & 19.064 & 2.125 \\
\hline & $(6.14)$ & $(1.61)$ & $(23.12)$ & $(13.55)$ & $(4.31)$ & $(1.54)$ & (11.19) & $(5.51)$ \\
\hline \multirow[t]{2}{*}{ inc1 } & 24.572 & 5.647 & 171.837 & 95.740 & -1.885 & -4.730 & -46.687 & -17.474 \\
\hline & $(24.93)$ & $(10.23)$ & $(93.92)$ & $(85.98)$ & $(17.49)$ & $(9.79)$ & $(45.46)$ & $(34.94)$ \\
\hline \multirow[t]{2}{*}{ inc2 } & -12.877 & -5.191 & $286.913^{*}$ & 70.338 & -24.577 & 4.806 & $-126.134^{*}$ & -24.868 \\
\hline & $(25.51)$ & (13.10) & (96.07) & $(110.14)$ & $(17.90)$ & (12.54) & $(46.50)$ & $(44.76)$ \\
\hline \multirow[t]{2}{*}{ inc3 } & -65.502 & 12.892 & 224.105 & 259.022 & 17.178 & 4.530 & -57.686 & -86.639 \\
\hline & $(61.65)$ & $(15.79)$ & $(232.23)$ & $(132.69)$ & $(43.26)$ & $(15.11)$ & $(112.40)$ & $(53.92)$ \\
\hline \multirow[t]{2}{*}{ inc4 } & 3.019 & 8.320 & 378.576 & 105.253 & 32.637 & 4.534 & -135.829 & -22.241 \\
\hline & $(44.22)$ & (18.09) & $(166.56)$ & (152.07) & (31.03) & $(17.32)$ & $(80.62)$ & $(61.80)$ \\
\hline \multirow[t]{2}{*}{ inc5 } & -20.782 & -6.848 & -238.868 & -35.520 & 33.993 & -15.250 & 99.298 & 10.549 \\
\hline & $(53.82)$ & $(13.57)$ & $(202.73)$ & (114.08) & $(37.76)$ & $(12.99)$ & $(98.13)$ & $(46.36)$ \\
\hline famwealth2 & -41.786 & -5.804 & 107.792 & 105.926 & -13.111 & $-17.532^{*}$ & -30.307 & -54.729 \\
\hline & $(29.30)$ & $(7.93)$ & $(110.38)$ & $(66.62)$ & $(20.56)$ & $(7.59)$ & $(53.43)$ & $(27.07)$ \\
\hline famwealth3 & -20.308 & 15.623 & $628.056^{*}$ & -22.535 & 14.103 & 17.009 & $-296.920^{*}$ & -3.485 \\
\hline & $(60.78)$ & $(14.78)$ & $(228.94)$ & $(124.20)$ & $(42.64)$ & $(14.14)$ & $(110.81)$ & $(50.48)$ \\
\hline mumocc1 & -6.223 & 9.705 & $293.923^{*}$ & 37.300 & 37.624 & -2.863 & -74.918 & -17.933 \\
\hline & $(33.23)$ & (10.01) & $(125.17)$ & $(84.14)$ & $(23.32)$ & (9.58) & $(60.59)$ & $(34.19)$ \\
\hline mumocc 2 & -10.150 & 0.191 & -96.509 & -13.709 & -1.971 & 7.848 & 54.114 & 20.013 \\
\hline & $(20.05)$ & $(10.50)$ & $(75.54)$ & $(88.22)$ & $(14.07)$ & (10.05) & $(36.56)$ & $(35.85)$ \\
\hline mumocc3 & -47.622 & 18.730 & -17.632 & 93.773 & -0.956 & -5.342 & 11.496 & -51.759 \\
\hline & $(35.96)$ & $(12.68)$ & $(135.46)$ & $(106.56)$ & $(25.23)$ & $(12.13)$ & $(65.57)$ & $(43.30)$ \\
\hline mumocc 4 & -2.318 & $43.830^{*}$ & 3.544 & 66.575 & -13.186 & 24.657 & 24.761 & -12.932 \\
\hline & $(30.94)$ & $(17.93)$ & $(116.56)$ & $(150.69)$ & $(21.71)$ & $(17.16)$ & $(56.42)$ & $(61.24)$ \\
\hline mumocc5 & -32.630 & 10.020 & -139.123 & 112.835 & -31.176 & $-22.936^{*}$ & 54.788 & $-70.256^{*}$ \\
\hline & $(30.24)$ & $(9.67)$ & $(113.89)$ & $(81.27)$ & $(21.21)$ & $(9.25)$ & $(55.12)$ & $(33.03)$ \\
\hline dadocc1 & 8.659 & -1.547 & 225.625 & -28.372 & 68.097 & -9.100 & -105.619 & 3.395 \\
\hline & $(54.14)$ & $(17.78)$ & $(203.92)$ & $(149.40)$ & $(37.98)$ & $(17.01)$ & $(98.70)$ & $(60.72)$ \\
\hline dadocc 2 & -14.584 & -18.363 & -60.861 & 12.561 & 54.342 & -17.582 & 6.198 & -20.774 \\
\hline & $(55.04)$ & $(18.66)$ & $(207.32)$ & $(156.82)$ & $(38.62)$ & $(17.86)$ & $(100.35)$ & $(63.73)$ \\
\hline dadocc3 & 41.166 & -2.045 & 430.312 & -135.350 & 81.581 & 13.913 & -173.906 & 54.339 \\
\hline & $(54.54)$ & $(19.61)$ & $(205.43)$ & $(164.80)$ & $(38.27)$ & $(18.77)$ & $(99.43)$ & $(66.97)$ \\
\hline dadocc 4 & 36.873 & 5.460 & 307.908 & -150.579 & 25.188 & 2.805 & -135.928 & 48.194 \\
\hline & $(46.88)$ & $(21.86)$ & $(176.60)$ & $(183.73)$ & $(32.89)$ & $(20.92)$ & $(85.48)$ & $(74.67)$ \\
\hline mumschool1 & -6.517 & -9.664 & -174.365 & 22.162 & -21.165 & -6.768 & 77.233 & -20.527 \\
\hline & $(23.75)$ & $(13.23)$ & $(89.48)$ & $(111.16)$ & $(16.67)$ & $(12.66)$ & $(43.31)$ & $(45.18)$ \\
\hline mumschool2 & 2.448 & 9.353 & 70.975 & 0.410 & -32.163 & -21.587 & -16.340 & -20.256 \\
\hline & $(30.14)$ & (11.19) & $(113.54)$ & $(94.05)$ & $(21.15)$ & $(10.71)$ & $(54.96)$ & $(38.22)$ \\
\hline mumschool3 & -21.605 & -1.030 & -27.896 & 86.766 & $-63.402^{*}$ & -4.704 & -7.963 & -49.210 \\
\hline & $(28.55)$ & $(10.73)$ & $(107.55)$ & $(90.18)$ & $(20.03)$ & $(10.27)$ & $(52.06)$ & $(36.65)$ \\
\hline dadschool1 & 7.200 & 27.144 & -15.346 & -26.957 & 26.663 & 4.797 & 30.516 & 29.078 \\
\hline & $(37.06)$ & $(13.80)$ & $(139.58)$ & $(115.96)$ & $(26.00)$ & $(13.20)$ & $(67.56)$ & $(47.12)$ \\
\hline dadschool2 & 38.002 & $35.750^{*}$ & -132.569 & -133.100 & 0.749 & $38.627^{* *}$ & 76.919 & 81.311 \\
\hline & $(26.15)$ & $(14.72)$ & $(98.49)$ & $(123.74)$ & $(18.34)$ & (14.09) & $(47.67)$ & $(50.29)$ \\
\hline dadschool3 & -3.321 & $24.457^{*}$ & -198.502 & -115.717 & 18.252 & 7.833 & 65.054 & 66.691 \\
\hline & $(31.07)$ & $(10.61)$ & $(117.02)$ & $(89.21)$ & $(21.80)$ & $(10.16)$ & $(56.64)$ & $(36.25)$ \\
\hline AdjR-sq & -0.119 & 0.047 & 0.511 & -0.159 & 0.210 & 0.249 & 0.392 & -0.114 \\
\hline Obs & 69 & 98 & 69 & 98 & 69 & 98 & 69 & 98 \\
\hline
\end{tabular}

${ }^{*} p<0.05,{ }^{* *} p<0.01,{ }^{* * *} p<0.001$. See text for dependent variable definitions. Samples in odd-numbered columns include participants in the final three experimental rounds; samples in evennumbered columns include participants in the pilot and first four experimental rounds. Samples sizes differ from those of Table 2 because both samples used in these regressions exclude all records whose survey data were incomplete. 
Table 3b: Correlates of Productivity Parameters: Other Covariates

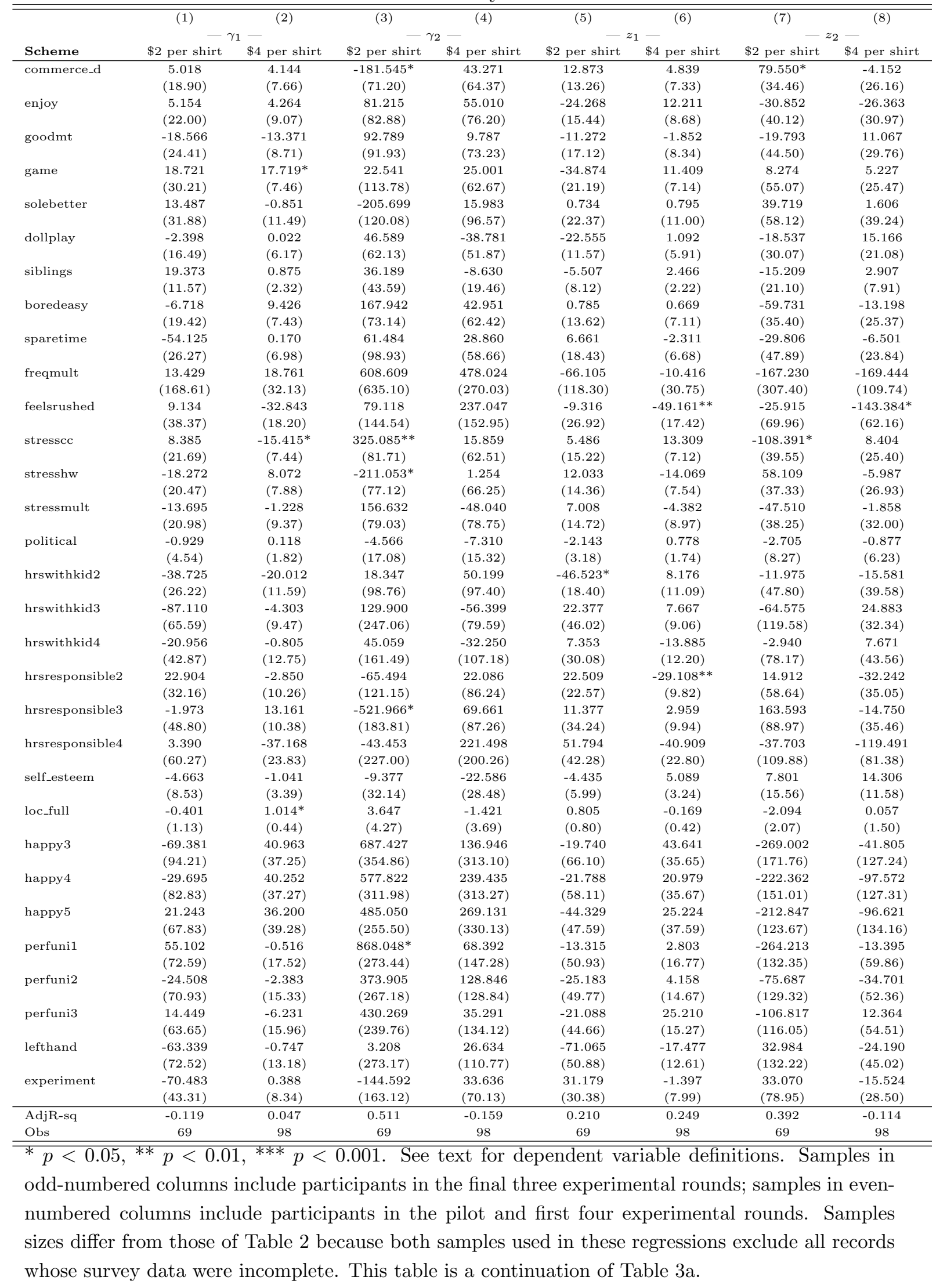


Table 4a: Correlates of Relative Productivity of Multitasking to Sole-tasking: Economic and Standard Demographic Covariates

\begin{tabular}{|c|c|c|c|c|}
\hline \multirow[b]{3}{*}{ Scheme } & \multirow{2}{*}{\multicolumn{2}{|c|}{${ }^{(1)}-\gamma_{2} / \gamma_{1}-(2)$}} & \multicolumn{2}{|r|}{ (4) } \\
\hline & & & \multicolumn{2}{|c|}{$-z_{2} / z_{1}-$} \\
\hline & $\$ 2$ per shirt & $\$ 4$ per shirt & $\$ 2$ per shirt & $\$ 4$ per shirt \\
\hline \multirow[t]{2}{*}{ female } & -0.138 & 0.009 & 0.012 & -0.083 \\
\hline & $(0.25)$ & $(0.18)$ & $(0.25)$ & $(0.13)$ \\
\hline \multirow[t]{2}{*}{ c_othasian } & $1.173^{*}$ & $0.503 *$ & $-1.034^{*}$ & -0.337 \\
\hline & $(0.45)$ & $(0.24)$ & $(0.44)$ & $(0.18)$ \\
\hline \multirow[t]{2}{*}{ c_panasian } & 0.642 & 0.106 & -0.233 & -0.138 \\
\hline & $(0.37)$ & $(0.25)$ & $(0.37)$ & $(0.18)$ \\
\hline \multirow[t]{2}{*}{ married } & $2.520^{*}$ & 1.456 & $-2.103^{*}$ & -1.023 \\
\hline & $(0.91)$ & $(0.85)$ & $(0.90)$ & $(0.62)$ \\
\hline \multirow[t]{2}{*}{ age } & -0.125 & -0.006 & 0.129 & 0.003 \\
\hline & $(0.07)$ & $(0.04)$ & $(0.07)$ & $(0.03)$ \\
\hline \multirow[t]{2}{*}{ inc1 } & 0.411 & 0.275 & -0.306 & -0.078 \\
\hline & $(0.28)$ & $(0.28)$ & $(0.28)$ & $(0.21)$ \\
\hline \multirow[t]{2}{*}{ inc2 } & $0.987 * *$ & 0.214 & $-0.722 *$ & -0.170 \\
\hline & $(0.29)$ & $(0.36)$ & $(0.28)$ & $(0.26)$ \\
\hline \multirow[t]{2}{*}{ inc3 } & 0.935 & 0.685 & -0.360 & -0.617 \\
\hline & $(0.70)$ & $(0.43)$ & $(0.69)$ & $(0.32)$ \\
\hline \multirow[t]{2}{*}{ inc4 } & $1.200^{*}$ & 0.273 & -1.079 & -0.244 \\
\hline & $(0.50)$ & $(0.50)$ & $(0.49)$ & $(0.36)$ \\
\hline \multirow[t]{2}{*}{ inc5 } & -0.646 & -0.067 & 0.382 & 0.124 \\
\hline & $(0.61)$ & $(0.37)$ & $(0.60)$ & $(0.27)$ \\
\hline \multirow[t]{2}{*}{ famwealth2 } & 0.455 & 0.348 & -0.049 & -0.247 \\
\hline & $(0.33)$ & $(0.22)$ & $(0.33)$ & $(0.16)$ \\
\hline \multirow[t]{2}{*}{ famwealth3 } & $2.031^{*}$ & -0.161 & $-1.937^{*}$ & -0.131 \\
\hline & $(0.69)$ & $(0.41)$ & $(0.68)$ & $(0.30)$ \\
\hline mumocc1 & $1.000 *$ & 0.035 & -0.574 & -0.105 \\
\hline & $(0.38)$ & $(0.27)$ & $(0.37)$ & $(0.20)$ \\
\hline mumocc 2 & -0.235 & -0.043 & 0.445 & 0.082 \\
\hline & $(0.23)$ & $(0.29)$ & $(0.22)$ & $(0.21)$ \\
\hline mumocc 3 & -0.013 & 0.173 & 0.211 & -0.287 \\
\hline & $(0.41)$ & $(0.35)$ & $(0.40)$ & $(0.26)$ \\
\hline mumocc 4 & -0.068 & -0.036 & 0.314 & -0.155 \\
\hline & $(0.35)$ & $(0.49)$ & $(0.34)$ & $(0.36)$ \\
\hline mumocc5 & -0.388 & 0.252 & 0.629 & -0.308 \\
\hline & $(0.34)$ & $(0.27)$ & $(0.34)$ & $(0.19)$ \\
\hline dadocc1 & 0.809 & -0.108 & -1.177 & 0.077 \\
\hline & $(0.61)$ & $(0.49)$ & $(0.60)$ & $(0.36)$ \\
\hline dadocc 2 & -0.108 & 0.107 & -0.346 & -0.061 \\
\hline & $(0.62)$ & $(0.51)$ & $(0.61)$ & $(0.38)$ \\
\hline dadocc3 & 1.313 & -0.402 & $-1.666^{*}$ & 0.283 \\
\hline & $(0.62)$ & $(0.54)$ & $(0.61)$ & $(0.39)$ \\
\hline dadocc 4 & 0.896 & -0.534 & -1.068 & 0.314 \\
\hline & $(0.53)$ & $(0.60)$ & $(0.52)$ & $(0.44)$ \\
\hline mumschool1 & -0.578 & 0.120 & $0.704 *$ & -0.120 \\
\hline & $(0.27)$ & $(0.36)$ & $(0.26)$ & $(0.27)$ \\
\hline mumschool2 & 0.120 & -0.076 & 0.165 & -0.018 \\
\hline & $(0.34)$ & $(0.31)$ & $(0.34)$ & $(0.23)$ \\
\hline mumschool3 & -0.094 & 0.279 & 0.323 & -0.305 \\
\hline & $(0.32)$ & $(0.29)$ & $(0.32)$ & $(0.22)$ \\
\hline dadschool1 & -0.003 & -0.215 & -0.005 & 0.184 \\
\hline & $(0.42)$ & $(0.38)$ & $(0.41)$ & $(0.28)$ \\
\hline dadschool2 & -0.543 & -0.607 & 0.474 & 0.366 \\
\hline & $(0.30)$ & $(0.40)$ & $(0.29)$ & $(0.30)$ \\
\hline dadschool3 & -0.573 & -0.483 & 0.225 & 0.418 \\
\hline & $(0.35)$ & $(0.29)$ & $(0.35)$ & $(0.21)$ \\
\hline AdjR-sq & 0.550 & -0.181 & 0.444 & -0.095 \\
\hline Obs & 69 & 98 & 69 & 98 \\
\hline
\end{tabular}

is identical to that applied to create Tables $3 \mathrm{a}$ and $3 \mathrm{~b}$. 
Table 4b: Correlates of Relative Productivity of Multitasking to Sole-tasking: Other Covariates

\begin{tabular}{|c|c|c|c|c|}
\hline \multirow[b]{3}{*}{ Scheme } & \multicolumn{2}{|c|}{ (1) } & \multicolumn{2}{|c|}{$(3)$} \\
\hline & \multicolumn{2}{|c|}{$-\gamma_{2} / \gamma_{1}-$} & \multicolumn{2}{|c|}{$-z_{2} / z_{1}-$} \\
\hline & $\$ 2$ per shirt & $\$ 4$ per shirt & $\$ 2$ per shirt & $\$ 4$ per shirt \\
\hline \multirow[t]{2}{*}{ commerce_d } & $-0.572^{*}$ & 0.112 & 0.436 & -0.068 \\
\hline & $(0.21)$ & $(0.21)$ & $(0.21)$ & $(0.15)$ \\
\hline \multirow[t]{2}{*}{ enjoy } & 0.130 & 0.124 & -0.091 & -0.259 \\
\hline & $(0.25)$ & $(0.25)$ & $(0.24)$ & $(0.18)$ \\
\hline \multirow[t]{2}{*}{ goodmt } & 0.360 & 0.127 & 0.011 & 0.061 \\
\hline & $(0.28)$ & $(0.24)$ & $(0.27)$ & $(0.18)$ \\
\hline \multirow[t]{2}{*}{ game } & -0.134 & -0.044 & 0.243 & -0.040 \\
\hline & $(0.34)$ & $(0.20)$ & $(0.34)$ & $(0.15)$ \\
\hline \multirow[t]{2}{*}{ solebetter } & -0.557 & 0.028 & 0.099 & 0.013 \\
\hline & $(0.36)$ & $(0.31)$ & $(0.35)$ & $(0.23)$ \\
\hline \multirow[t]{2}{*}{ dollplay } & 0.024 & -0.119 & 0.062 & 0.076 \\
\hline & $(0.19)$ & $(0.17)$ & $(0.18)$ & $(0.12)$ \\
\hline \multirow[t]{2}{*}{ siblings } & 0.022 & -0.029 & -0.067 & 0.002 \\
\hline & $(0.13)$ & $(0.06)$ & $(0.13)$ & $(0.05)$ \\
\hline \multirow[t]{2}{*}{ boredeasy } & $0.562^{*}$ & 0.068 & -0.321 & -0.091 \\
\hline & $(0.22)$ & $(0.20)$ & $(0.22)$ & $(0.15)$ \\
\hline sparetime & 0.454 & 0.093 & -0.199 & 0.003 \\
\hline & $(0.30)$ & $(0.19)$ & $(0.29)$ & $(0.14)$ \\
\hline freqmult & 1.491 & 1.359 & -0.132 & -1.041 \\
\hline & $(1.91)$ & $(0.88)$ & $(1.88)$ & $(0.65)$ \\
\hline feelsrushed & 0.260 & 0.857 & -0.132 & -0.719 \\
\hline & $(0.43)$ & $(0.50)$ & $(0.43)$ & $(0.37)$ \\
\hline stresscc & $0.962^{* *}$ & 0.144 & $-0.616^{*}$ & -0.018 \\
\hline & $(0.25)$ & $(0.20)$ & $(0.24)$ & $(0.15)$ \\
\hline stresshw & -0.530 & -0.046 & 0.281 & 0.080 \\
\hline & $(0.23)$ & $(0.22)$ & $(0.23)$ & $(0.16)$ \\
\hline stressmult & $0.607^{*}$ & -0.154 & -0.368 & -0.004 \\
\hline & $(0.24)$ & $(0.26)$ & $(0.23)$ & $(0.19)$ \\
\hline political & 0.003 & -0.025 & -0.025 & -0.008 \\
\hline & $(0.05)$ & $(0.05)$ & $(0.05)$ & $(0.04)$ \\
\hline hrswithkid2 & 0.084 & 0.283 & 0.195 & -0.128 \\
\hline & $(0.30)$ & $(0.32)$ & $(0.29)$ & $(0.23)$ \\
\hline hrswithkid3 & 0.766 & -0.157 & -0.389 & 0.179 \\
\hline & $(0.74)$ & $(0.26)$ & $(0.73)$ & $(0.19)$ \\
\hline hrswithkid4 & 0.174 & -0.094 & 0.008 & 0.195 \\
\hline & $(0.48)$ & $(0.35)$ & $(0.48)$ & $(0.26)$ \\
\hline hrsresponsible 2 & -0.181 & 0.062 & -0.118 & -0.072 \\
\hline & $(0.36)$ & $(0.28)$ & $(0.36)$ & $(0.21)$ \\
\hline hrsresponsible 3 & $-1.475^{*}$ & 0.152 & 0.804 & -0.147 \\
\hline & $(0.55)$ & $(0.28)$ & $(0.54)$ & $(0.21)$ \\
\hline hrsresponsible 4 & 0.251 & 0.868 & -0.622 & -0.612 \\
\hline & $(0.68)$ & $(0.65)$ & $(0.67)$ & $(0.48)$ \\
\hline self_esteem & -0.052 & -0.050 & 0.106 & 0.062 \\
\hline & $(0.10)$ & $(0.09)$ & $(0.09)$ & $(0.07)$ \\
\hline loc_full & 0.018 & -0.012 & -0.020 & 0.000 \\
\hline & $(0.01)$ & $(0.01)$ & $(0.01)$ & $(0.01)$ \\
\hline happy3 & 2.174 & 0.078 & -1.184 & -0.630 \\
\hline & $(1.06)$ & $(1.02)$ & $(1.05)$ & $(0.75)$ \\
\hline happy 4 & 1.726 & 0.388 & -0.926 & -0.845 \\
\hline & $(0.94)$ & $(1.02)$ & $(0.92)$ & $(0.75)$ \\
\hline happy5 & 1.277 & 0.481 & -0.915 & -0.830 \\
\hline & $(0.77)$ & $(1.08)$ & $(0.75)$ & $(0.79)$ \\
\hline perfuni1 & $2.290^{*}$ & 0.202 & -1.405 & -0.152 \\
\hline & $(0.82)$ & $(0.48)$ & $(0.81)$ & $(0.35)$ \\
\hline perfuni2 & 0.933 & 0.437 & -0.055 & -0.269 \\
\hline & $(0.80)$ & $(0.42)$ & $(0.79)$ & $(0.31)$ \\
\hline perfuni3 & 0.976 & 0.190 & -0.331 & -0.066 \\
\hline & $(0.72)$ & $(0.44)$ & $(0.71)$ & $(0.32)$ \\
\hline lefthand & -0.157 & 0.085 & 0.829 & -0.083 \\
\hline & $(0.82)$ & $(0.36)$ & $(0.81)$ & $(0.27)$ \\
\hline experiment & -0.101 & 0.090 & 0.071 & -0.092 \\
\hline & $(0.49)$ & $(0.23)$ & $(0.48)$ & $(0.17)$ \\
\hline AdjR-sq & 0.550 & -0.181 & 0.444 & -0.095 \\
\hline Obs & 69 & 98 & 69 & 98 \\
\hline
\end{tabular}

${ }^{*} p<0.05,{ }^{* *} p<0.01,{ }^{* * *} p<0.001$. Sample selection is identical to that applied to create Tables $3 \mathrm{a}$ and $3 \mathrm{~b}$. This table is a continuation of Table $4 \mathrm{a}$ 


\subsection{Productivity responses to multitasking and incentives}

We now proceed to estimating average task-specific productivity losses due to multitasking, together with the own- and cross- price elasticities of task performance.

\subsubsection{Which is better: Sequential sole-tasking, or multitasking?}

We first use our results on productivity to ask the following question, for each participant: If a window of time were available in which s/he could either sequentially sole-task (spending half the time in each task) or multitask for the entire time, which would be better for him/her to choose, purely from a productivity standpoint?

Figure 5 provides the graphical answer to this question, where the analysis is conducted separately by incentive scheme. The left-hand graph shows results for the $\$ 2$-per-shirt incentive scheme, and the right-hand graph shows results for the $\$ 4$-per-shirt incentive scheme. The vertical axis is the percentage of participants for whom a particular result applies, where the denominator of this percentage is the total number of participants who faced the given compensation scheme during their 'non-learning' phases.

Our results indicate that, for the vast majority of people, multitasking is preferable for at least one task in terms of the total output produced. Sequential sole-tasking is almost never preferable-except for a very small number of participants in the $\$ 2$-per-shirt incentive scheme. We also see that the change to a higher relative payoff for housework induces a change in the distribution of optimal time allocations for the two tasks, in the expected direction. Specifically, the largest group of participants in the low-payoff regime would generate higher baby care productivity under the multitasking allocation and higher housework productivity under the sequential sole-tasking regime. Once we pay more per shirt, the gap narrows between this group of participants and the next-highest group, for whom sequential sole-tasking is preferable from a baby-care productivity standpoint, but multitasking is preferable from a housework standpoint. Essentially, this group size shift illustrates the exertion of more effort to increase the multitasking productivity of housework, in order to capitalize on the increased economic incentives for shirts correctly sorted, and less effort on the baby care task.

\subsubsection{Elasticities}

Finally, we move to a calculation of aggregate elasticity figures. To obtain these figures, we first calculate average products for each use of time for each good under each payoff regime and stage-order structure. As before, a participant's total productivity in a baby- 


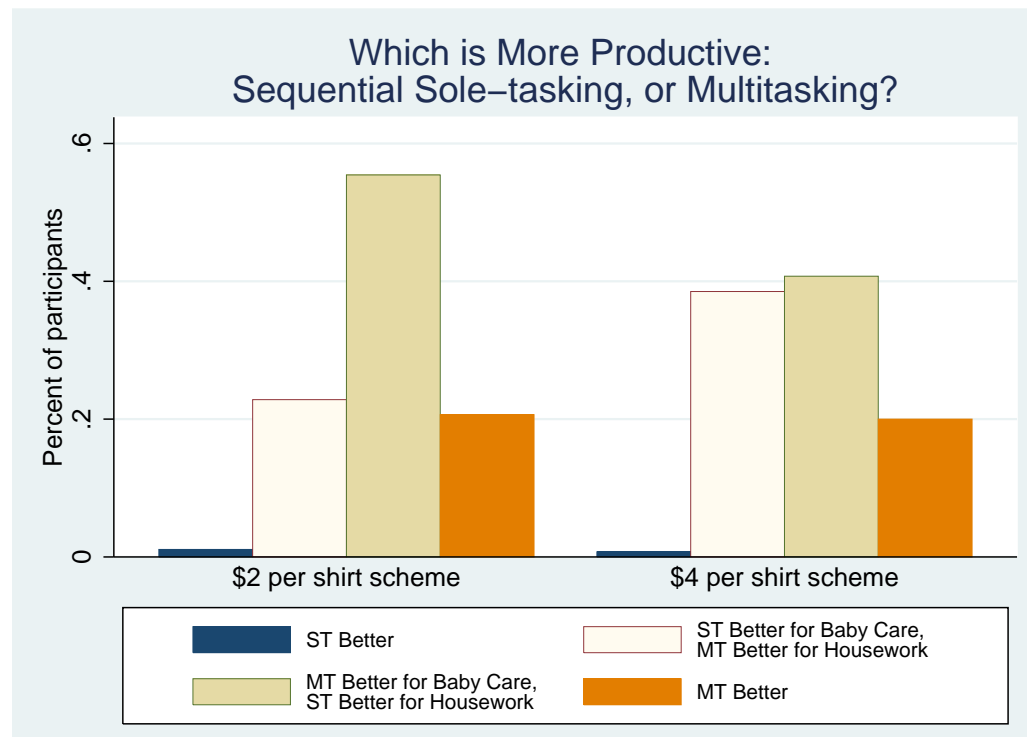

Figure 5: $\$ 2 /$ shirt scheme (left) versus $\$ 4 /$ shirt scheme (right)

care task is calculated as the total money payoff the participant earned in that task. To construct the participant's average product per minute, we divide this figure by the number of minutes spent in the stage in which that payoff was earned for that task (three minutes if sole-tasked and six minutes if multitasked). Average product in the clothes-sorting task is calculated as the number of shirts sorted correctly minus the number of shirts sorted incorrectly, divided by the number of minutes spent in the stage in which that task was undertaken.

Panel A of Table 5 shows average products in the sole-tasking stages for the baby-care and clothes-sorting tasks, for each payoff regime and stage-order structure. Panel B of Table 5 shows similar figures for the multitasking stages. All figures represent means and standard deviations across the entire cell of experimental participants. Bolded figures in Table 5 represent those means that are plausibly uncontaminated by any learning effects, as they are calculated using only the second instances of the clothes-sorting task. Only these figures are used in calculating the relative productivity measures discussed below. ${ }^{6}$

The figures in Table 5 imply that the loss in baby-care average product due to moving

\footnotetext{
${ }^{6}$ The magnitude of the learning effect, calculated by comparing mean average product in the first soletasked clothes-sorting task that participants faced to the second one (roughly 47 and 55 respectively, as shown in Table 5), is roughly 8 shirts per minute. Again, it is evident that there are no learning effects for baby care.
} 
from a sole-tasked to multitasked environment is between $29 \%$ and $40 \%$, depending on the level of remuneration offered in the clothes-sorting task that competed for the participant's effort in the multitasking stage. ${ }^{7}$ Not surprisingly, the more valuable the alternative task (sorting clothes) was to participants, the more that average per-minute product in the baby-care task fell in moving from the sole-tasked to the multitasked environment.

For sorting clothes, the figures in Table 5 imply that moving from a sole-tasking to a multitasking environment results in a loss in average product of between $36 \%$ and $46 \%$, again depending upon the relative remuneration available in the two tasks competing for participants' effort in the multitasking context.

These average product losses are far greater than those found by Buser \& Peter (2012). However, in addition to the fact that our study investigates very different tasks than theirs, our design features bona fide multitasking: participants face two tasks that are simultaneously competing for their attention, while the design of Buser \& Peter (2012) presents tasks in sequence but with different (enforced versus optional) switching regimes.

Using the numbers in Table 5, we can compute rough estimates of the relevant own- and cross-price elasticities of task supply for baby care and sorting clothes. When we double the opportunity cost of baby care, by moving from the low-payoff regime to the high-payoff regime, baby-care average product in the multitasked setting decreases by $16.84 \%$, whereas average product in the clothes-sorting task increases by $19.71 \%$. The first of these figures translates into an own-price output elasticity of -0.1685 for the baby care task within the multitasking setting, while the second translates into a cross-price output elasticity of 0.1971 .

In the opposite direction, moving from the $\$ 4$ per shirt scheme to the $\$ 2$ per shirt scheme doubles the opportunity cost of sorting clothes. This change then brings about a decrease in the clothes-sorting average product of $16.46 \%$, with a concomitant increase in baby-care average product of $20.25 \%$. Hence, the own-price supply elasticity for clothessorting is roughly -.1646, very similar to that of baby care. The estimate of the cross-price supply elasticity of baby care is .2025, also very similar to that of clothes sorting. These results indicate that economic incentives are clearly at work with respect to multitasking. Participants are willing to perform less baby care in a multitasked setting if they are

\footnotetext{
${ }^{7}$ As mentioned above when discussing the histogram of baby care productivity parameters, it should be noted that approximately $64 \%$ of participants earned the maximum amount possible in the sole-tasked baby care stage. This may mean that the average product losses estimated in this section are underestimates. However, re-calculating the figures above using only those participants who did not earn the maximum in the sole-tasked baby care stage yields slightly smaller, but still fairly similar, estimated losses for the baby care task: $23 \%$ and $32 \%$ in the low-payoff and high-payoff regimes respectively.
} 
Table 5: Mean Average Products

\begin{tabular}{|c|c|c|c|c|}
\hline \multicolumn{5}{|l|}{ Panel $A$} \\
\hline Task: & \multicolumn{2}{|c|}{$\begin{array}{l}\text { Sole-tasked } \\
\text { baby care }\end{array}$} & $\begin{array}{c}\text { Sole-tasked } \\
\text { clothes sorting: } \\
\text { Low-payoff regime }\end{array}$ & $\begin{array}{c}\text { Sole-tasked } \\
\text { clothes sorting: } \\
\text { High-payoff regime }\end{array}$ \\
\hline $\begin{array}{l}\text { Structure } 1(\mathrm{~N}=124) \\
\text { (low-payoff stages } \\
\text { presented first) }\end{array}$ & \multicolumn{2}{|c|}{$\begin{array}{l}\mathbf{1 1 7 . 4 4} \\
(10.67)\end{array}$} & $\begin{array}{c}47.17 \\
(10.10)\end{array}$ & $\begin{array}{l}\mathbf{5 5 . 7 5} \\
(8.47)\end{array}$ \\
\hline $\begin{array}{l}\text { Structure } 2(\mathrm{~N}=88) \\
\text { (high-payoff stages } \\
\text { presented first) }\end{array}$ & \multicolumn{2}{|c|}{$\begin{array}{c}\mathbf{1 1 8 . 4 2} \\
(8.34)\end{array}$} & $\begin{array}{l}\mathbf{5 5 . 0 8} \\
(7.85)\end{array}$ & $\begin{array}{l}47.85 \\
(8.81)\end{array}$ \\
\hline \multicolumn{5}{|l|}{ Panel $B$} \\
\hline Task: & $\begin{array}{c}\text { Multitasked } \\
\text { baby care: } \\
\text { Low-payoff regime }\end{array}$ & $\begin{array}{c}\text { Multitasked } \\
\text { baby care: } \\
\text { High-payoff regime }\end{array}$ & $\begin{array}{c}\text { Multitasked } \\
\text { clothes sorting: } \\
\text { Low-payoff regime }\end{array}$ & $\begin{array}{c}\text { Multitasked } \\
\text { clothes sorting: } \\
\text { High-payoff regime }\end{array}$ \\
\hline $\begin{array}{l}\text { Structure } 1(\mathrm{~N}=124) \\
\text { (low-payoff stages } \\
\text { presented first) }\end{array}$ & $\begin{array}{c}96.85 \\
(32.72)\end{array}$ & $\begin{array}{l}\mathbf{6 9 . 9 6} \\
(45.15)\end{array}$ & $\begin{array}{c}23.82 \\
(13.95)\end{array}$ & $\begin{array}{c}\mathbf{3 5 . 7 8} \\
(19.52)\end{array}$ \\
\hline $\begin{array}{l}\text { Structure } 2(\mathrm{~N}=88) \\
\text { (high-payoff stages } \\
\text { presented first) }\end{array}$ & $\begin{array}{c}\mathbf{8 4 . 1 3} \\
(42.74)\end{array}$ & $\begin{array}{c}80.24 \\
(40.90)\end{array}$ & $\begin{array}{c}\mathbf{2 9 . 8 9} \\
(18.50)\end{array}$ & $\begin{array}{c}30.80 \\
(16.24)\end{array}$ \\
\hline
\end{tabular}

Average product is calculated for baby care as experimental dollars earned per minute, given the remuneration scheme for this task; see text for details. Average product for sorting clothes is the number of correctly-sorted shirts minus the number of incorrectly-sorted shirts, per minute. Standard deviations are in parentheses. Bolded figures are those from which learning effects are credibly excluded. Participants earned two experimental dollars per shirt in the 'low-payoff regime' and four experimental dollars per shirt in the 'high-payoff regime'. See text for full descriptions of experimental treatments. 
compensated for it, which is accomplished in our experiment through increased pay for the alternative task of sorting clothes.

\section{Conclusion}

Our paper provides the first economic estimates of the relative productivity of multitasked time to sole-tasked time in individuals' production of child and household goods, using a novel experimental approach. Our results show raw output reductions associated with multitasking that are far greater than those estimated in prior experimental work. We believe this is due to the different tasks that we analyze and the different approach that we take in our experimental design compared to that taken in prior work.

In our experiment we represent a change in preferences by a change in the relative monetary payoffs of the clothes-sorting and baby-care tasks. Using this approach, we are able to calculate own- and cross-price elasticities of supply for sorting clothes and baby care. Our results suggest, at a minimum, the order of magnitude of a change in multitasking performance for which differences across people in these utility parameters may be responsible. They also suggest that the influence of preferences or habits may be able to be meaningfully moderated through the application of targeted economic incentives. 


\section{Appendix}

\subsection{Questionnaire}

The following questions/statements were posed to participants before the income-earning stages of the experiment. The statements were followed by scaled answer alternatives, ranging from Strongly Agree to Strongly Disagree, or Always to Never. Double-asterisked items form part of the modified Rosenberg (1965) self-esteem battery; single-asterisked items form part of the modified Rotter (1966) locus of control battery:

- I get bored easily.

- I think I'm good at multitasking.

- I enjoy being busy.

- Things get done better when you focus on doing only one thing at a time, without getting distracted.

- On the whole, I am satisfied with myself.**

- At times I think I am no good at all.**

- I feel that I have a number of good qualities.**

- I am able to do things as well as most people.**

- I feel I do not have much to be proud of.**

- I certainly feel useless at times.**

- I feel that I am a person of worth, or at least on an equal plane with others. ${ }^{* *}$

- I wish I could have more respect for myself.**

- All in all, I am inclined to feel that I am a failure.**

- I take a positive attitude toward myself.**

- How often do you feel rushed or pressed for time?

- How often do you have spare time that you don't know what to do with?

- How often do you play computer games or video games?

- When you are at home, how often do you find yourself doing more than one thing at once? 
- When you were a child, how often did you play caretaking games with dolls and/or stuffed animals?

- I have little control over the things that happen to me.*

- There is really no way I can solve some of the problems I have.*

- There is little I can do to change many of the important things in my life.*

- I often feel helpless in dealing with the problems of life.*

- Sometimes I feel that I'm being pushed around in life.*

- What happens to me in the future mostly depends on me.*

- I can do just about anything I really set my mind to.*

The following questions were posed to participants after the income-earning stages of the experiment:

- How stressful did you find the multitasking stages of the experiment?

- How stressful did you find the sole-tasking clothes-sorting stages of the experiment?

- How stressful did you find the sole-tasking baby-care stage of the experiment?

- What is your year of birth?

- What is your month and day of birth?

- Please indicate your gender.

- Please type in your nationality/citizenship.

- Please type in the country in which you were born.

- Please type in the country whose culture you identify with most strongly.

- Please type in the postcode of the area you live in.

- Please type in the name of the UNSW degree program in which you are enrolled, if any (Bachelor of Economics; Bachelor of Commerce; Masters of Commerce; etc.).

- When do you expect to graduate (month and year)?

- Are you a UNSW student?

- Are you an international student? 
- Have you ever participated in an experiment before?

- What is your personal weekly after-tax income from all sources?

- Do you speak English at home?

- What is your current living situation?

- Are you currently...(married, in a partnership, or single).

- Are you a parent?

- How many siblings did you grow up with (in the same household?

- How many hours in your adult life (over 14 yrs old) have you spent awake in your home in the presence of an infant under 1 year old?

- How many hours in your life have you been completely personally responsible for the care of an infant under 1 year old?

- How many people under age 15 are you currently living with (in the same household?

- How many women aged 15 or older are living in your household (including yourself, if applicable)? [Only asked of participants who are living with children.]

- How many parents of children under age 15 who are living in your household also live in your household (including yourself, if applicable)? [Only asked of participants who are living with children.]

- How many people aged 15 or older are living in your household and are NOT parents of children under 15 living in your household (including yourself, if applicable)? [Only asked of participants who are living with children.]

- Please type in the age of the youngest child in your household. [Only asked of participants who are living with children.]

- What is the highest year of school you have completed?

- How much schooling did your mother complete?

- How much schooling did your father complete?

- Did your mother complete an educational qualification after leaving school? Please include any trade certificates, apprenticeships, diplomas, degrees or other educational qualifications.

- If yes, from where was her highest level qualification obtained? 
- Did your father complete an educational qualification after leaving school? Please include any trade certificates, apprenticeships, diplomas, degrees or other educational qualifications.

- If yes, from where was his highest level qualification obtained?

- Please select the category of class of professions your mother's occupation falls into.

- Please select the category of class of professions your father's occupation falls into.

- Which hand do you write with?

- In political matters, people talk of "the left" and "the right". How would you place your views on this scale, generally speaking?

- All things considered in your life, how happy would you say you are usually?

- Would you say that your family is ... (wealthier, the same, or poorer than others)?

- Overall, how would you rate your performance at university? 


\subsection{Hard-copy instructions to participants}

Figures 6 (front) and 7 (back) are reproductions of the contents of the instruction sheet presented to participants when they entered the ASBLab to participate in this experiment.

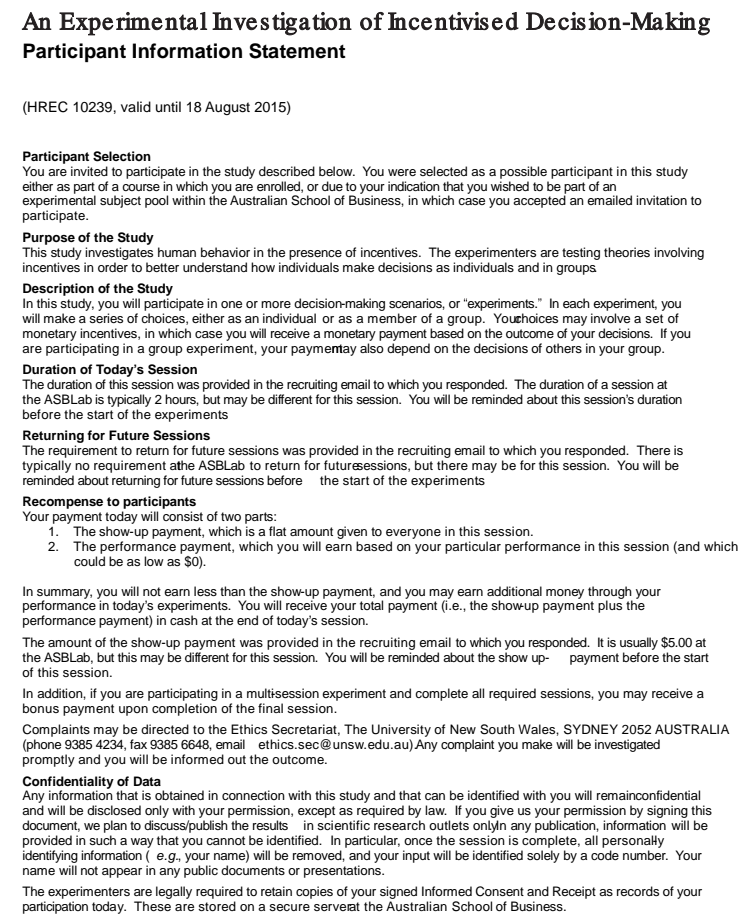

Figure 6: Front page of hard-copy instructions to participants 


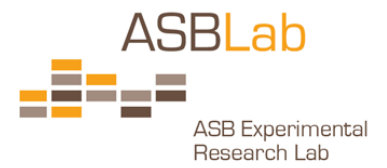

Risks

There are no anticipated risks attending your participation in this study.

Benefits

There are no anticipated benefits attending your participation in this study.

\section{Your consent}

Your decision whether or not to participate will not prejudice your future relations with the University of New South Wales

the Australian School of Business, or the experimenters. If you decide to participate, you are free to withdraw your

consent and to discontinue participation at any time without prejudic e. If you wish to withdraw during this session, please

raise your hand. An experimenter will come to your station, and you can then quietly inform him or her of your intention

to withdraw.

You can keep the show-up payment if you decide to withdraw. How ever, you will not be paid the performance payment unless you complete the entire session. In addition, if you are participating in a multisession experiment, you will not be paid the multi-session bonus.

If you have any questions, please feel free to ask us. If you have any additional questions later, either the ASB Lab

Director (listed below) or the Chief Investigator listed on the Consent Form will be happy to answer them.

You will be given a copy of this form to keep.

Participation Restrictions

If you meet any of the following criteria, you are not eligible to participate in today's session:

$\mathrm{z}$ You are under 18 years of age.
$\mathrm{z}$ You are in a position of authority over or authority under one of the experimenters in this session (e.g., an

employer or an employee of one of the experimenters).

$\mathrm{z}$ You are a current student of one of today's experimenters.

If any of these apply to you, please inform an experimenter before this session begins.

ASBLab Contact

Questions about the purpose or methodology of this study should be directed to theASBLab Director:

Dr Ben Greiner

Australian School of Business

University of New South Wales

UNSW Sydney, NSW 2052 AUSTRALIA

Email:bgreiner@unsw.edu.au

AUSTRALIAN

SCHOOL OF BUSINESS

THE UNIVERSTY OF NEW SOUTH WALES

Figure 7: Back page of hard-copy instructions to participants 


\subsection{On-screen instructions to participants}

The following instructions were presented on-screen to participants commencing the experiment.

Introduction

This is an experiment about decisionmaking and performance in multi-tasked performance settings. You will earn real money through your participation in this experiment, and your payment will depend on your performance. Please follow the instructions carefully. If you have a question, please raise your hand and an experiment administrator will come to you.

During the experiment, any talking or other communication between participants is forbidden. Please TURN OFF your mobile phone and other electronic devices now.

Your performance in this experiment is anonymous, and you are identified solely by your participant number. The payment you will receive at the end of the experiment will be kept confidential from all other participants.

This experiment will proceed in several stages. In some stages, you will see pictures of babies or clothing, and to earn money you will need to click on or drag-and-drop icons. Before each stage you will see on-screen instructions that explain exactly what to do in that stage, and how you will be paid out for your performance.

You will do the following things, in this order:

- Answer a few questions about yourself.

- Participate in 3 minutes of sole-tasked baby care.

- Participate in 3 minutes of sole-tasked clothes-sorting.

- Participate in 3 minutes of sole-tasked clothes-sorting, with a different compensation structure than the previous stage.

- Participate in 6 minutes of multi-tasked baby care and clothes-sorting.

- Participate in 6 minutes of multi-tasked baby care and clothes-sorting, with a different compensation structure than the previous stage.

- Complete a demographics questionnaire while we prepare your payout.

All compensation is described in terms of experimental dollars. Each experimental dollar that you earn during the experiment will be converted into a fixed fraction of an Australian dollar. Your final payout at the end of the experiment will be the sum of TWO (2) of the three sole-tasked 
stages plus ONE (1) of the two multi-tasked stages. The actual stages for which you are paid out will be chosen randomly by the computer.

Please put on your headphones now. Thank you for your participation. We hope you enjoy this experiment! If you have any questions, please raise your hand and an experiment administrator will come to you. 


\section{References}

Abraham, K. \& Mackie, C., eds (2005), Beyond the Market: Designing Nonmarket Accounts for the United States, The National Academies Press, Washington, D.C.

Becker, G. (1965), 'A theory of the allocation of time', The Economic Journal 75, 493-517.

Buser, T. \& Peter, N. (2012), 'Multitasking', Experimental Economics .

Floro, M. S. \& Miles, M. (2003), 'Time use, work and overlapping activities: Evidence from Australia', Cambridge Journal of Economics 27, 881-904.

Folbre, N. (2006), 'Measuring care: Gender, empowerment, and the care economy', Journal of Human Development 7(2), 183-199.

Folbre, N. \& Yoon, J. (2007), 'What is child care? Lessons from time use surveys of major English-Speaking countries', Review of Economics of the Household 5(3).

Folbre, N., Yoon, J., Finnoff, K. \& Fuligni, A. S. (2005), 'By what measure?: Family time devoted to children in the United States', Demography 42(2), 373-390.

Greiner, B. (2004), Forschung und wissenschaftliches rechnen 2003, chapter An Online Recruitment System for Economic Experiments, pp. 79-93.

Kalenkoski, C. M. \& Foster, G. (2008), 'The quality of time spent with children in Australian households', Review of Economics of the Household 6, 243-266.

Rosenberg, M. (1965), Society and The Adolescent Self-Image, Princeton University Press.

Rotter, J. (1966), 'Generalized expectancies for internal versus external control of reinforcement', Psychological Monographs $\mathbf{8 0 .}$.

Rubinstein, J., Meyer, D. E. \& Evans, J. E. (2001), 'Executive control of cognitive proceese in task switching', Journal of Experimental Psychology: Human Perception and Performance 27(4), 763-797. 